\title{
Morphological characteristics, chemical and genetic diversity of kenaf (Hibiscus cannabinus L.) genotypes
}

\author{
Jaihyunk Ryu - Soon-Jae Kwon • Dong-Gun Kim - Min-Kyu Lee $\cdot$ Jung Min Kim • Yeong Deuk Jo • \\ Sang Hoon Kim $\cdot$ Sang Wook Jeong $\cdot$ Kyung-Yun Kang $\cdot$ Se Won Kim $\cdot$ Jin-Baek Kim $\cdot$ Si-Yong Kang
}

Received: 16 October 2017 / Revised: 9 November 2017 / Accepted: 9 November 2017

(c) Korean Society for Plant Biotechnology

\begin{abstract}
The kenaf plant is used widely as food and in traditional folk medicine. This study evaluated the morphological characteristics, functional compounds, and genetic diversity of 32 kenaf cultivars from a worldwide collection. We found significant differences in the functional compounds of leaves from all cultivars, including differences in levels of chlorogenic acid isomer (CAI), chlorogenic acid (CA), kaempferol glucosyl rhamnoside isomer (KGRI), kaempferol rhamnosyl xyloside (KRX), kaemperitrin (KAPT) and total phenols (TPC). The highest TPC, KAPT, CA, and KRX contents were observed in the $\mathrm{C} 22$ cultivars. A significant correlation was observed between flowering time and DM yield, seed yield, and four phenolic compounds (KGRI, KRX, CAI, and TPC) $(\mathrm{P}<0.01)$. To assess genetic diversity, we used 80 simple sequence repeats (SSR) primer sets and identified 225 polymorphic loci in the kenaf cultivars. The polymorphism information content and genetic diversity values ranged from 0.11 to 0.79 and 12 to 0.83 , with average values of 0.39 and 0.43 , respectively. The cluster analysis of the SSR markers showed that the kenaf genotypes could be clearly divided into three clusters based on flowering time. Correlations analysis was conducted for the 80 SSR markers; morphological, chemical and growth traits were found for 15 marker traits (corolla, vein, petal, leaf, stem color, leaf shape, and KGRI content) with significant marker-trait correl-
\end{abstract}

J. Ryu, S.-J. Kwon ( $₫)$, D.-G. Kim, M.-K. Lee, J. M. Kim, Y. D. Jo, S. H. Kim, S. W. Jeong, K.-Y Kang, S. W. Kim, J.-B. Kim, S.-Y. Kang $(\square)$

Advanced Radiation Technology Institute, Korea Atomic Energy

Research Institute, Jeongup, Jeonbuk 56212, Korea

E-mail: soonjaekwon@kaeri.re.kr, sykang@kaeri.re.kr

S. W. Jeong

Jangheung Research Institute for Mushroom Industry, Jangheung 59338, Korea

\section{K.-Y. Kang}

Suncheon Research Center for Natural Medicines, Suncheon, Republic of Korea ations. These results could be used for the selection of kenaf cultivars with improved yield and functional compounds.

Keywords Kenaf, Morphological chracteristics, Phenolic compounds, SSR markers, Correlation analysis

$\begin{array}{ll}\text { Abbreviations } \\ \text { SSR } & \text { Simple sequence repeats } \\ \text { UPLC } & \text { Ultra-high performance liquid chromatography } \\ \text { CAI } & \text { Chlorogenic acid isomer } \\ \text { CA } & \text { Chlorogenic acid } \\ \text { KGRI } & \text { Kaempferol glucosyl rhamnoside isomer } \\ \text { KRX } & \text { Kaempferol rhamnosyl xyloside } \\ \text { KAPT } & \text { Kaemperitrin } \\ \text { TPC } & \text { Total phenols }\end{array}$

\section{Introduction}

Kenaf (Hibiscus cannabinus L.), an annual herbaceous crop of the family Malvaceae $(2 n=36)$, is a short-day annual herbaceous plant that tolerates a broad range of soil types and climates (Dempsey 1975). Kenaf has traditionally been used to make rope but the plant is used in many different ways, including as pulp, potting media, a bioplastic, and cellulosic biofuel (Alexopoulou et al. 2013). Recently, kenaf being used valuable dual-purpose crop for fiber and medicinal. Its leaves contain large amounts of a variety of compounds including polyphenols (Jin et al. 2013; Ryu et al. 2017a). The most common phenolic compounds in kenaf are the kaempferol glycoside, caffeic acid, myricetin glycoside, and $p$-hydroxybenzoic acid (Ryu et al. 2017a). In ayurvedic medicine, the kenaf are used for bilious, blood, diabetes, biliousness, coughs and throat disorders (Alexopoulou et al. 2013; Ryu et al. 2017a).

Kenaf has erect, tall plants with few or no branches that 
may reach $3 \sim 5 \mathrm{~m}$ in height depending on climatic conditions (Alexopoulou et al. 2013; Ryu et al. 2013). Its leaves may be non-lobed (entire), shallow-lobed, or deeply lobed (palmate). Kenaf flowers, are bell-shaped, open with five petals, and have colors ranging from light cream to dark purple (Alexopoulou et al. 2013). Korean Kenaf cultivars are divided into three maturation groups depending on flowering date; earlymaturing, mid-late maturing, and late maturing. Early-maturing groups mature in $70 \sim 80$ days after seeding and it allow seed harvests, but the lower biomass. Late-maturing groups grow vegetatively for $130 \sim 140$ days and yield significantly higher biomass. However, late maturation reduces seed quality (Ryu et al. 2016a; Jeong et al. 2017). Breeding selection of new kenaf cultivars is the most effective measure to increase biomass and seed yields per unit area (Alexopoulou et al. 2013). Mutation breeding has the merits of creating new mutant characteristics and adding only very few traits without disturbing the other characteristics of a cultivar (Kang et al. 2016; Ryu et al., 2017b). Mutagenic agents, such as radiation, can be used to induce mutations and generate genetic variants from which desired mutants may be selected (Visser et al. 1971). This process offers the possibility of inducing desirable attributes that either have not developed in nature or have been lost during evolution. In crops, mutagenesis has already been used to introduce many useful traits affecting plant size, blooming time or color, and resistance to pathogens (IAEA 2016; Kang et al. 2016; Ryu et al. 2016b; Ryu et al. 2017b).

To cultivate kenaf in a tropical environment, it is necessary to evaluate the available genotypes in terms of their morphological characters (Faruq et al. 2013). Moreover, the identification of genetic relationships and diversity is one of the most important factors in the selection of cultivars for a breeding program (Jeong et al. 2017; Thakur et al. 2017). Simple sequence repeats (SSRs) are one of the most commonly used types of DNA molecular markers for revealing genetic diversity among genotypes (Varshney et al. 2005). Knowledge of level of genetic diversity, morphological and chemical relationships among genotypes essential for establishing, managing, and ensuring the long-term success of crop-improvement programs (Varshney et al. 2005; Jeong et al. 2017; Thakur et al. 2017). In this study, we compared morphological, phytochemical, and genetic characteristics among kenaf cultivars from eight countries (Bangladesh, China, India, Iran, Italy, Korea, USA, and Russia). We examined morphological and chemical characteristics to establish relationships, and compared these relationships to genetic relationships. In addition, we used SSR markers to evaluate genetic diversity, and demonstrated significant associations between the SSR markers and morphological and phytochemical characteristics.

\section{Materials and Methods}

\section{Plant materials}

Thirty-two cultivars were studied. These included six mutant genotypes (Jangdae, Jeokbong, RS1, RS2, WFM1-2 and Bae$\mathrm{kma}$ ), two original cultivars (Jinju and C14), and 24 accessions collected worldwide from the Genebank of Rural Development Administration (RDA) in Korea and the Bangladesh Jute Mills Corporation (BJC). The Jangdae cultivar combines high biomass and seed yield and has been tested by the Korea Seed and Verity Service (KSVS). Five of the morphological mutants exhibiting changes in flower color (Baekma, WFM1-2) and stem color (Jeokbong, RS1, RS2) were derived from the same kenaf cultivar (C14) introduced from Italy. The whole plant and leaves of the genotypes were harvested at the flowering time of each genotype for dry matter (DM) yield and phenolic compounds analysis. Seeds were planted in plots $(3 \times 4.2 \mathrm{~m})$ with row spacings of 20 and $60 \mathrm{~cm}$, respectively. Fertilizer (N:P:K 4:2:2 w/w/w) was applied at $550 \mathrm{~kg} /$ ha shortly after seeding. Manure was spread before planting, but the plants were not fertilized after planting. The experiment was conducted at the Korea Atomic Energy Research Institute.

Morphological and agronomic characteristics

All genotypes were planted on mid-May and measured for morphological and agronomic characteristics during 2012 to 2015. The cultivars were divided into three different flowering groups, early flowering ( $70 \sim 84$ day after seeding), mid-late flowering (101 134 day after seeding), and non-flowering. We measured the morphological traits of each sampled individual: leaf shape (palmate 1, entire 2), leaf color (green 1 , purple 2), branch color (green 1, brown 2, purple 3), vein color (green 1, purple 2), hypocotyl colors (green 1, purple 2), stem colors (brown 1, green 2, purple 3) corolla color (green 1, purple 2, non 3), and petal colors (Ivory 1, white 2, non 3).

UPLC analysis

Phenolic compounds were analyzed using a ultra-high performance liquid chromatography (UPLC) system (CBM-20A, Shimadzu Co., Kyoto, Japan) with two gradient pump systems 
(LC-30AD, Shimadzu), a UV-detector (SPD-MB0A, Shimadzu), an auto sample injector (SIL-30AC, Shimadzu), and a column oven (CTO-30A, Shimadzu). Separation was achieved on an XR-ODS column $(3.0 \times 100 \mathrm{~mm}, 1.8 \mu \mathrm{m}$, Shimadzu, Japan) using a linear gradient elution program with a mobile phase containing solvent $\mathrm{A}(0.1 \%$, v/v, trifluoroacetic acid in distilled deionized water) and solvent $\mathrm{B}(0.1 \%, \mathrm{v} / \mathrm{v}$, trifluoroacetic acid in acetonitrile). Samples for UPLC analysis of phenolic compound contents were ground using a grinder immediately prior to analysis. All samples were ground to achieve a particle size that would pass through a $500 \mathrm{~mL}$ sieve. For UPLC analysis, ground samples $(1 \mathrm{~g})$ were extracted in $5 \mathrm{~mL}$ water for $16 \mathrm{~h}$ and filtrated through a 0.45 $\mu \mathrm{m}$ membrane filter. The phenolic compounds were separated using the following gradient: $0 \sim 5 \mathrm{~min}, 10 \sim 15 \% \mathrm{~B} ; 5 \sim 10$ min, $15 \sim 20 \% \mathrm{~B} ; 10 \sim 15 \mathrm{~min}, 20 \sim 30 \% \mathrm{~B} ; 15 \sim 20 \mathrm{~min}$, $30 \sim 50 \% \mathrm{~B} ; 20 \sim 25 \mathrm{~min}, 50 \sim 75 \% \mathrm{~B} ; 25 \sim 30 \mathrm{~min}, 75 \sim$ $100 \% \mathrm{~B} ; 30 \sim 32 \mathrm{~min}, 100 \sim 5 \% \mathrm{~B} ; 32 \sim 35 \mathrm{~min}, 5 \sim 0 \%$ B. The phenolic compounds were detected at $280 \mathrm{~nm}$. Identification of kaempferitrin (KAPT) was determined based on the retention times of commercial standards (UV spectrum) and the Chlorogenic acid isomer (CAI), Chlorogenic acid (CA), Kaempferol glucosyl rhamnoside isomer (KGRI), Kaempferol rhamnosyl xyloside (KRX) were identified using retention time, UV-visible spectral characteristics.

DNA extraction and simple sequence repeat analysis

DNA was extracted from young leaves of each cultivar using the cetyltrimethylammonium bromide (CTAB) method. A total of 70 EST-SSR primers set were synthesized based on published sequence information (Jeong et al. 2017) and 10 genomic SSR primer sets were obtained from the US National Center for Biotechnology Information (NCBI, http:// www.ncbi.nlm.nih.gov/) Hibiscus cannabinus database. SSR sequence PCR reactions were carried out in $25 \mu \mathrm{L}$ of a mixture containing $20 \mathrm{ng}$ genomic DNA, 10 pmol primer, 1 unit Taq polymerase, $10 \times$ PCR reaction buffer, and 0.2 $\mathrm{mM}$ dNTP. The PCR cycling conditions were as follows: $94^{\circ} \mathrm{C}$ for $5 \mathrm{~min}$ (initial denaturation), followed by 40 cycles at $94^{\circ} \mathrm{C}$ for $45 \mathrm{~s}$ (predenaturation), $55^{\circ} \mathrm{C}$ for $45 \mathrm{~s}$ (annealing), $72^{\circ} \mathrm{C}$ for $90 \mathrm{~s}$ (extension), with a final $7 \mathrm{~min}$ extension at $72^{\circ} \mathrm{C}$ and cooling to $4^{\circ} \mathrm{C}$. The samples were fractionated using a LabChip GX electrophoresis system (Caliper Life Science, USA).

Statistical analysis

The chemical analysis data were subjected to analysis of variance using a multiple comparisons method with the statistical software package SPSS version 12 (SPSS Institute, USA). Differences were determined to be significant at $p<$ 0.05 . When the treatment effect was significant, means were separated using Duncan's multiple range tests. The correlation coefficients between the morphological and agronomics characteristics and the phenolic compound contents were obtained using Pearson's correlation coefficients $(P<0.01)$ to examine the degree among kenaf genotypes.

All of the SSR bands were scored as 0 or 1 for the absence or presence of the band, respectively. The polymorphism information content (PIC), mean gene diversity (GD), and Shannon's information index (SI) for each SSR marker were calculated using Power Marker Ver. 3.25.

The morphological, chemical and genetic dendrogram were constructed using the phylogenetic tree using the Neighbor Joining method (Saitou and Nei 1987) with the statistical software package Power Marker Ver. 3.25.

We determined correlation between morphological characteristics, phenolic compound contents and SSR markers using TASSEL 3.0.1 software (Bradbury et al. 2007). We performed three tests for significance. First, the Q general linear model (GLM) was used on the chosen Q-matrix derived from STRUCTURE with 10,000 permutations to test marker significance and determine the experiment-wise $\mathrm{P}$ value for each marker's significance. Second, the Q-mixed linear model (Q-MLM) method was used to determine a kinship matrix. SPAGeDi was used to calculate kinship (K) coefficients.

\section{Results}

Morphological and agronomic characteristics

Selected results of the evaluation of morphological characteristics are presented in Table 1 and Figure 1. The leaf shapes of the kenaf cultivars were divided into two types: 11 cultivars with entire and 21 cultivars with palmate leaves. All kenaf cultivars had green leaves, except for the Jeokbong cultivar, which had greenish purple leaves. The colors of branches and stems were green, brown, or purple. Veins, hypocotyls, and corollas were green or purple. Finally, flower petals were ivory (18 genotypes) or white (2 genotypes), while 12 genotypes were non-flowering.

The growth characteristics of the kenaf genotypes are shown in Table 2. We observed significant differences for most of the growth and yield characteristics including plant height, fresh mater yield (FM) and seed yield. The plant 
Table 1 The origin and morphological characteristics of kenaf genotypes used in this study

\begin{tabular}{|c|c|c|c|c|c|c|c|c|c|c|c|c|}
\hline No. & Name & Origin & $\begin{array}{c}\text { Source } \\
\text { (cultivar or } \\
\text { accession } \\
\text { number) }\end{array}$ & Flowering & $\begin{array}{l}\text { Leaf } \\
\text { shape }\end{array}$ & $\begin{array}{l}\text { Leaf } \\
\text { color }\end{array}$ & Branch & Vein & Hypocotyl & $\begin{array}{l}\text { Stem } \\
\text { color }\end{array}$ & Corolla & $\begin{array}{l}\text { Petal } \\
\text { color }\end{array}$ \\
\hline 1 & C9 & Russia & IT202789 & Early & Palmate & Green & Green & Green & Purple & Brown & Green & Ivory \\
\hline 2 & $\mathrm{C} 10$ & India & IT202790 & Early & Palmate & Green & Green & Green & Green & Brown & Green & Ivory \\
\hline 3 & $\mathrm{C} 11$ & Iran & IT202791 & Early & Entire & Green & Brown & Green & Purple & Brown & Green & Ivory \\
\hline 4 & $\mathrm{C} 12$ & Italy & IT202792 & Early & Palmate & Green & Brown & Green & Purple & Brown & Green & Ivory \\
\hline 5 & $\mathrm{C} 13$ & Russia & IT202793 & Early & Palmate & Green & Brown & Green & Purple & Brown & Green & Ivory \\
\hline 6 & $\mathrm{C} 14$ & Italy & IT202794 & Early & Palmate & Green & Brown & Green & Purple & Brown & Green & Ivory \\
\hline 7 & $\mathrm{C} 15$ & Africa & IT202795 & Early & Palmate & Green & Green & Green & Purple & Brown & Green & Ivory \\
\hline 8 & $\mathrm{C} 16$ & China & IT202796 & Early & Palmate & Green & Green & Green & Green & Brown & Green & Ivory \\
\hline 9 & $\mathrm{C} 17$ & China & IT202797 & Early & Palmate & Green & Green & Green & Purple & Brown & Green & Ivory \\
\hline 10 & $\mathrm{C} 18$ & China & IT202798 & Early & Palmate & Green & Brown & Green & Green & Brown & Green & Ivory \\
\hline 11 & $\mathrm{C} 19$ & India & II202799 & Early & Palmate & Green & Brown & Green & Purple & Brown & Green & Ivory \\
\hline 12 & $\mathrm{C} 20$ & India & IT202800 & Early & Palmate & Green & Brown & Green & Green & Brown & Green & Ivory \\
\hline 13 & $\mathrm{C} 22$ & Russia & IT202802 & Early & Palmate & Green & Brown & Green & Purple & Brown & Green & Ivory \\
\hline 14 & $\mathrm{RS} 1$ & $\mathrm{C} 14$ & Mutant line & Early & Entire & Green & Purple & Purple & Purple & Puple & Purple & Ivory \\
\hline 15 & $\mathrm{RS} 2$ & $\mathrm{C} 14$ & Mutant line & Early & Palmate & Green & Purple & Purple & Purple & Puple & Purple & Ivory \\
\hline 16 & Jeokbong & $\mathrm{C} 14$ & Cultivar & Early & Entire & Purple & Purple & Purple & Purple & Puple & Purple & Ivory \\
\hline 17 & Baekma & $\mathrm{C} 14$ & Cultivar & Early & Entire & Green & Green & Green & Green & Green & Green & White \\
\hline 18 & WFM1-2 & $\mathrm{C} 14$ & Mutant line & Early & Entire & Green & Brown & Green & Purple & Brown & Green & White \\
\hline 19 & Jangdae & Jinju & Cultivar & Mid-late & Palmate & Green & Green & Green & Green & Green & Green & Ivory \\
\hline 20 & Jinju & Unknown & Accession & Mid-late & Entire & Green & Green & Green & Green & Green & Green & Ivory \\
\hline 21 & Hongma300 & China & Cultivar & Late & Palmate & Green & Brown & Green & Green & Brown & $-{ }^{x}$ & Ivory \\
\hline 22 & Hongma74-3 & China & Cultivar & Late & Palmate & Green & Green & Green & Purple & Green & - & Ivory \\
\hline 23 & Everglades41 & USA & Cultivar & Late & Palmate & Green & Brown & Green & Purple & Brown & - & Ivory \\
\hline 24 & ACC3748 & Unknown & $\mathrm{BJC}^{\mathrm{y}}$ & Late & Entire & Green & Brown & Green & Green & Brown & - & Ivory \\
\hline 25 & ACC4111 & Unknown & $\mathrm{BJC}$ & Late & Entire & Green & Brown & Green & Green & Brown & - & Ivory \\
\hline 26 & ACC4153 & Unknown & $\mathrm{BJC}$ & Late & Entire & Green & Brown & Green & Green & Brown & - & Ivory \\
\hline 27 & ACC4139 & Unknown & BJC & Late & Palmate & Green & Brown & Green & Green & Brown & - & Ivory \\
\hline 28 & ACC4443 & Unknown & $\mathrm{BJC}$ & Late & Palmate & Green & Purple & Purple & Purple & Purple & - & Ivory \\
\hline 29 & ACC4751 & Unknown & BJC & Late & Entire & Green & Brown & Green & Green & Brown & - & Ivory \\
\hline 30 & ACC4985 & Unknown & $\mathrm{BJC}$ & Late & Palmate & Green & Green & Green & Green & Green & - & Ivory \\
\hline 31 & ACC5014 & Unknown & BJC & Late & Palmate & Green & Green & Green & Purple & Green & - & Ivory \\
\hline 32 & ACC5072 & Unknown & BJC & Late & Entire & Green & Green & Green & Green & Green & - & Ivory \\
\hline
\end{tabular}

${ }^{\mathrm{z}}$ IT No. : Genebank, National Agro Biodiversity Center, Rural Development Administration, ${ }^{\mathrm{y}}$ BJC: Bangladesh Jute Mills Corporation xon-flowering

height of all the genotypes ranged from 234.4 to 415.9 $\mathrm{cm}$. The Hongma300 had the highest FM yield and lowest yield was found in C20 genotype. The highest seed yield was recorded for the Jangdae cultivar and twelve late flowering genotypes were impossible seed harvest.

We generated a phylogenetic tree using the Neighbor Joining method (Saitou and Nei 1987) for morphological and agronomic characteristics relationships (Fig. 2A). The keanf genotypes were divided into six groups based on phylogenetic tree the morphological and agronomic characteristics. Group 1 consisted of two genotypes (C10 and C16) with concurrent flowering date and morphological phenotype (palmate and green leaf, green vein, coroll and hypocotyl color, brown stem, ivory flower). Group 2 contained three 


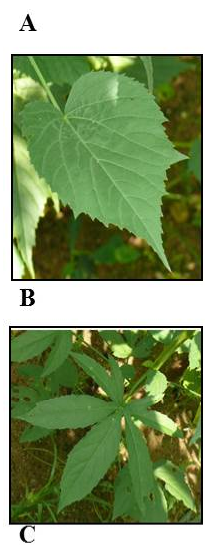

D

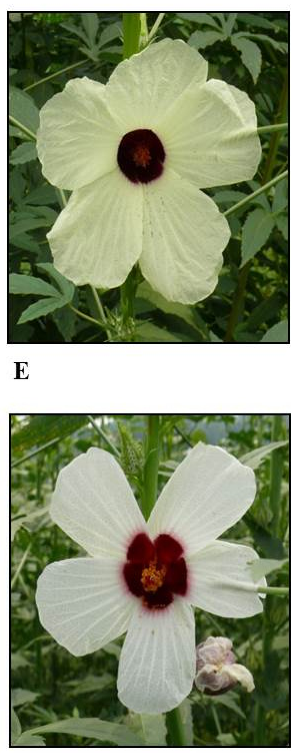

F

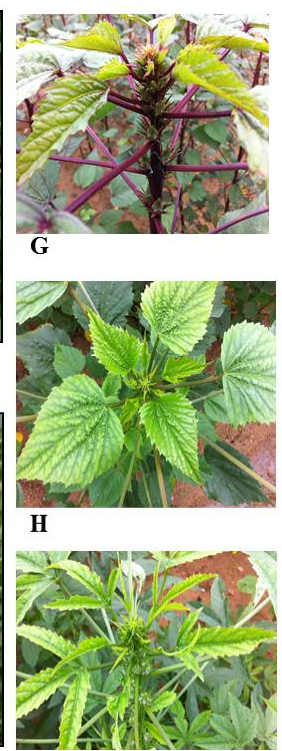

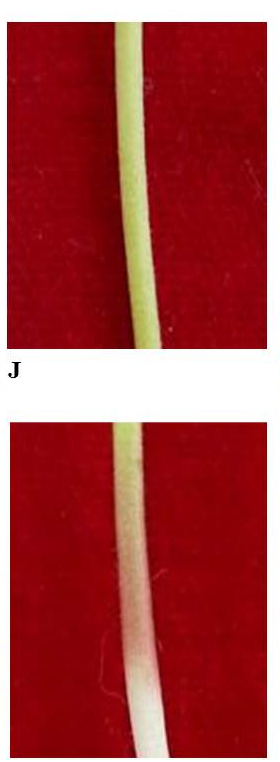
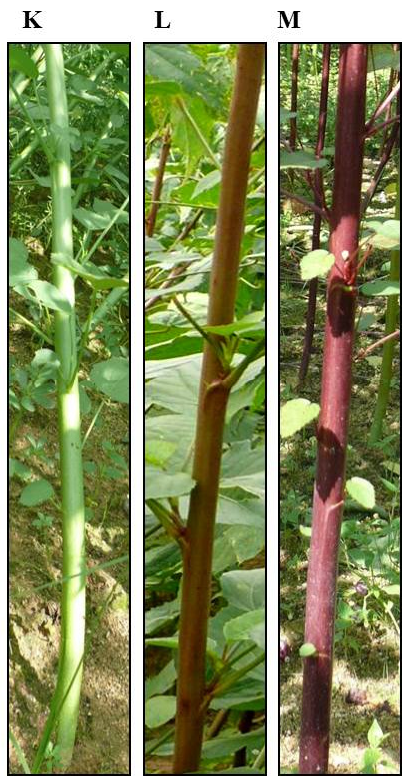

Fig. 1 Comparison of morphological characteristics of kenaf genotypes. A: Entire leaf, B: palmate leaf, C: leaf color (purple), D: Ivory petal, E: white petal, F: branch color (purple), G: branch color (brown), H: branch color (green), I: Hypocotyl color (green), J: Hypocotyl Color (purple), K: stem color (green), L: stem color (brown), M: stem color (purple)

Table 2 Growth characteristics of kenaf genotypes

\begin{tabular}{|c|c|c|c|}
\hline Genotype & $\begin{array}{l}\text { Plant height } \\
(\mathrm{cm})\end{array}$ & $\begin{array}{l}\text { Dry matter yield } \\
\text { (ton/ha) }\end{array}$ & $\begin{array}{c}\text { Seed yield } \\
(\mathrm{kg} / \mathrm{ha})\end{array}$ \\
\hline C9 & $247.5 \pm 05.2^{\mathrm{k}}$ & $74.8 \pm 1.6^{\mathrm{h}}$ & $588.7 \pm 06.0^{f}$ \\
\hline $\mathrm{C} 10$ & $238.8 \pm 08.7^{1}$ & $75.3 \pm 2.7^{\mathrm{h}}$ & $514.2 \pm 09.2^{\mathrm{j}}$ \\
\hline C11 & $274.5 \pm 03.6^{\mathrm{gh}}$ & $77.7 \pm 1.1^{\mathrm{gh}}$ & $636.5 \pm 04.3^{\mathrm{e}}$ \\
\hline $\mathrm{C} 12$ & $272.5 \pm 01.6^{\mathrm{h}}$ & $76.0 \pm 0.5^{\text {gh }}$ & $652.3 \pm 02.1^{\mathrm{d}}$ \\
\hline C13 & $252.7 \pm 01.7^{\mathrm{k}}$ & $74.0 \pm 0.5^{\mathrm{h}}$ & $538.5 \pm 01.7^{\mathrm{hi}}$ \\
\hline $\mathrm{C} 14$ & $268.1 \pm 02.9^{\mathrm{h}}$ & $76.0 \pm 0.8^{\mathrm{gh}}$ & $641.0 \pm 03.3^{\mathrm{de}}$ \\
\hline $\mathrm{C} 15$ & $276.9 \pm 02.9^{\mathrm{gh}}$ & $76.7 \pm 0.8^{\mathrm{gh}}$ & $669.1 \pm 03.3^{\mathrm{c}}$ \\
\hline $\mathrm{C} 16$ & $257.7 \pm 16.6^{\mathrm{j}}$ & $71.9 \pm 4.6^{\mathrm{hi}}$ & $545.1 \pm 17.9^{\mathrm{h}}$ \\
\hline $\mathrm{C} 17$ & $254.3 \pm 17.4^{\mathrm{j}}$ & $71.9 \pm 5.0^{\mathrm{hi}}$ & $566.2 \pm 20.3^{\mathrm{g}}$ \\
\hline $\mathrm{C} 18$ & $253.6 \pm 24.6^{\mathrm{j}}$ & $69.9 \pm 6.8^{\mathrm{hij}}$ & $457.1 \pm 23.4^{\mathrm{k}}$ \\
\hline $\mathrm{C} 19$ & $231.2 \pm 10.2^{1}$ & $68.4 \pm 3.0^{\mathrm{hij}}$ & $426.9 \pm 09.4^{1}$ \\
\hline $\mathrm{C} 20$ & $248.2 \pm 11.8^{\mathrm{k}}$ & $63.6 \pm 3.4^{\mathrm{j}}$ & $412.1 \pm 09.3^{\mathrm{m}}$ \\
\hline $\mathrm{C} 22$ & $234.4 \pm 05.6^{1}$ & $65.9 \pm 1.5^{\mathrm{j}}$ & $325.1 \pm 03.7^{\mathrm{n}}$ \\
\hline RS1 & $261.6 \pm 00.4^{\mathrm{i}}$ & $70.2 \pm 0.0^{\mathrm{hi}}$ & $536.9 \pm 00.2^{\mathrm{hi}}$ \\
\hline $\mathrm{RS} 2$ & $255.8 \pm 03.8^{\mathrm{j}}$ & $69.4 \pm 1.0^{\mathrm{hij}}$ & $529.1 \pm 03.8^{\mathrm{i}}$ \\
\hline Jeokbong & $268.9 \pm 09.3^{\mathrm{h}}$ & $73.1 \pm 2.5^{\mathrm{h}}$ & $579.5 \pm 09.8^{\mathrm{f}}$ \\
\hline Baekma & $288.4 \pm 01.1^{\mathrm{fg}}$ & $80.7 \pm 0.3^{\mathrm{g}}$ & $686.8 \pm 01.2^{\mathrm{b}}$ \\
\hline WFM1-2 & $293.3 \pm 02.0^{f}$ & $80.7 \pm 0.6^{\mathrm{g}}$ & $687.0 \pm 02.4^{\mathrm{b}}$ \\
\hline Jangdae & $345.8 \pm 11.8^{\text {de }}$ & $117.0 \pm 4.0^{\mathrm{f}}$ & $847.5 \pm 14.2^{\mathrm{a}}$ \\
\hline Jinju & $350.5 \pm 08.9^{\text {de }}$ & $125.7 \pm 3.2^{\mathrm{e}}$ & $180.0 \pm 02.2^{\circ}$ \\
\hline Hongma300 & $406.2 \pm 03.4^{\mathrm{ab}}$ & $144.1 \pm 1.2^{\mathrm{ab}}$ & $0.0^{\mathrm{p}}$ \\
\hline Hongma74-3 & $394.3 \pm 02.1^{\mathrm{b}}$ & $139.9 \pm 0.8^{\mathrm{bc}}$ & $0.0^{\mathrm{p}}$ \\
\hline Everglades41 & $415.9 \pm 03.7^{\mathrm{a}}$ & $148.1 \pm 1.3^{\mathrm{a}}$ & $0.0^{\mathrm{p}}$ \\
\hline ACC 3748 & $369.5 \pm 02.8^{\mathrm{c}}$ & $133.6 \pm 1.0^{\mathrm{d}}$ & $0.0^{\mathrm{p}}$ \\
\hline ACC4111 & $352.4 \pm 08.9^{\mathrm{d}}$ & $139.9 \pm 3.5^{\mathrm{bc}}$ & $0.0^{\mathrm{p}}$ \\
\hline ACC4153 & $357.7 \pm 02.9^{\mathrm{cd}}$ & $138.6 \pm 3.3^{\mathrm{c}}$ & $0.0^{\mathrm{p}}$ \\
\hline ACC4139 & $385.4 \pm 03.6^{\mathrm{b}}$ & $134.6 \pm 1.2^{\mathrm{cd}}$ & $0.0^{\mathrm{p}}$ \\
\hline ACC4443 & $390.1 \pm 02.4^{\mathrm{b}}$ & $133.1 \pm 0.8^{\mathrm{d}}$ & $0.0^{\mathrm{p}}$ \\
\hline ACC4751 & $382.4 \pm 03.4^{\mathrm{bc}}$ & $136.0 \pm 1.2^{\mathrm{c}}$ & $0.0^{\mathrm{p}}$ \\
\hline ACC4985 & $338.2 \pm 05.9^{\mathrm{e}}$ & $135.9 \pm 2.3^{\mathrm{c}}$ & $0.0^{\mathrm{p}}$ \\
\hline ACC 5014 & $365.6 \pm 08.6^{\mathrm{cd}}$ & $138.8 \pm 3.3^{\mathrm{c}}$ & $0.0^{\mathrm{p}}$ \\
\hline ACC5072 & $352.5 \pm 03.0^{\mathrm{d}}$ & $138.1 \pm 1.2^{\mathrm{c}}$ & $0.0^{\mathrm{p}}$ \\
\hline
\end{tabular}

The letters above each point indicate a significant difference at the $5 \%$ level (Duncan's multiple range tests, $n=3$ ) 
$\mathbf{A}$

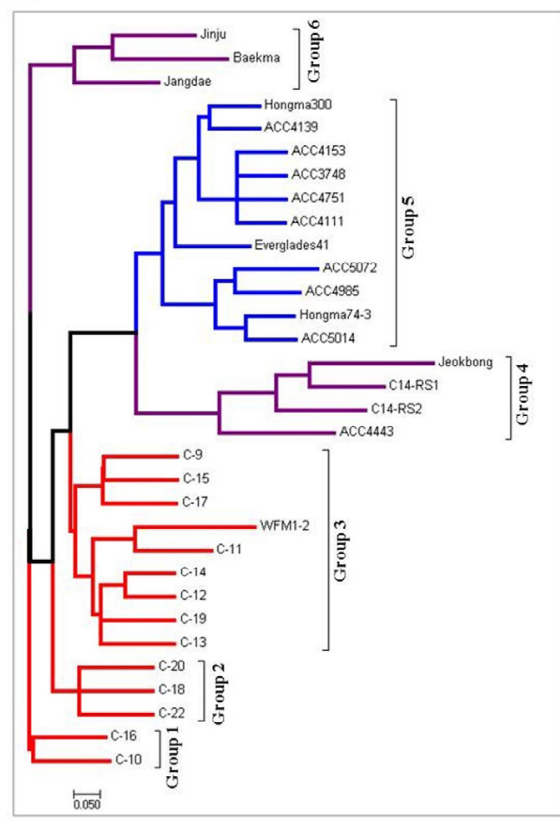

B

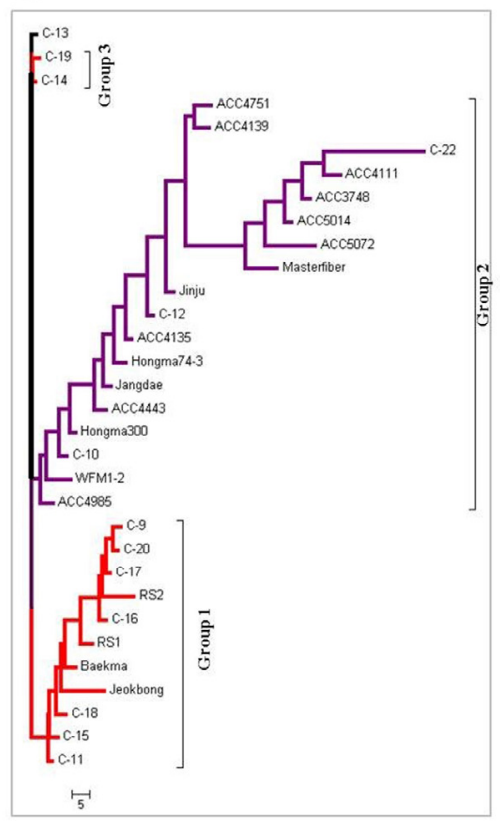

C

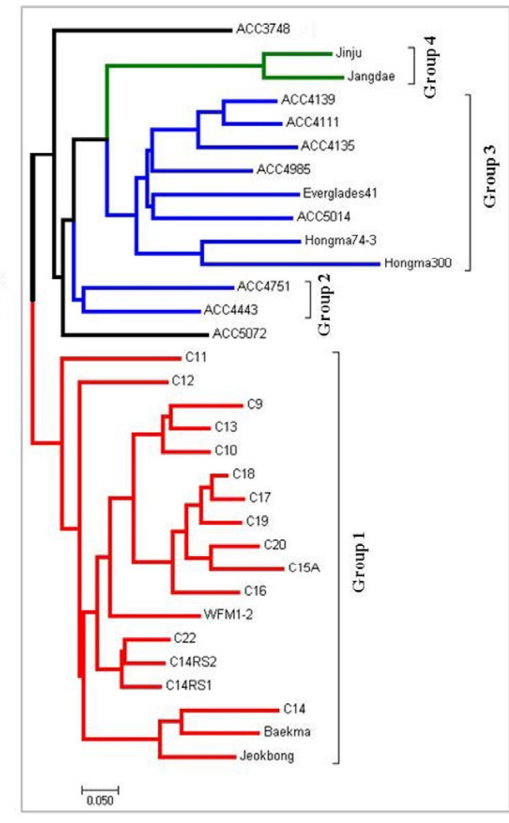

Early flowering group $\longrightarrow$ Non flowering group

Mid-late flowering group

Independent group

Fig. 2 Dendrogram showing the phenotypic relationship among the kenaf genotypes based on Pearson's correlation coefficients generated by morphological traits

genotypes ( $\mathrm{C} 18, \mathrm{C} 20$ and $\mathrm{C} 22)$ with same flowering date, leaf shape, leaf, branch, vein, stem, corolla and petal colors. Group 3 contained 9 early flowering genotypes (C9, C11, C12, C13, C14, C15, C17, C19 and WFM1-2). Group 5 included three purple stem mutant (Jeokbong, RS1 and RS2) and ACC4443 genotypes. Group 5 consists of 11 late flowering genotypes (Hongma300, Hongma74-3, Everglades41, ACC3748, ACC4111, ACC4153, ACC4139, ACC4751, ACC4985, ACC5014 and ACC5072). Group 6 included two mutant cultivars (Jangdae and Baekma) and Jinju with leaf, branch, vein, hypocotyl, stem and collroa were all green.

Phenolic compounds

The phenolic compound contents of the kenaf cultivars are shown in Table 3 and Figure 3. Five compounds, chlorogenic acid isomer (CAI), chlorogenic acid (CA), kaempferol glucosyl rhamnoside isomer (KGRI), kaempferol rhamnosyl xyloside (KRX), kaemperitrin (KAPT) were isolated. Significant differences in phenolic compound contents were observed among cultivars. The CAI content for all genotypes ranged from 18.0 to $64.0 \mathrm{mg} / 100 \mathrm{~g}$. The ACC5072 genotypes had contained higher levels of CAI compounds than other genotypes. The highest CA content was observed in C22 (153.8 mg/100 g), and lowest levels were found in C20 $(64.4 \mathrm{mg} / 100 \mathrm{~g})$. The KGRI levels of all genotypes ranged from $29.8 \mathrm{mg} / 100 \mathrm{~g}$ for C9 to $108.6 \mathrm{mg} / 100 \mathrm{~g}$ for ACC4111. The KRX contents ranged from 8.7 to $\mathrm{C} 22 \mathrm{mg} / 100 \mathrm{~g}$, with the highest amounts being in the C22. The KAPT content ranged from 97.8 to $306.1 \mathrm{mg} / 100 \mathrm{~g}$. The highest KAPT content was observed in cultivar $\mathrm{C} 22$, and the lowest values were found in C9 (Fig. 3). The total polyphenol content (TPC) differed significantly among the cultivars, with the highest level found in C22 (766.8 mg/100 g $\left.{ }^{-1}\right)$. The C9 cultivar had the lowest content (317.0 mg/100 g $\mathrm{g}^{-1}$ ).

Analysis of the phylogenetic tree showed that the 32 kenaf genotypes divided into three groups with the exception of the $\mathrm{C} 13$ genotypes, based on the six phenolic compounds (Fig. 2B). Group 1 included eleven early flowering genotypes (C9, C11, C15, C16, C17, C18, C20, Jeokbong, RS1, RS2 and Baekma). Group 2 included four early flowering genotypes (C10, C12, C22 and WFM1-2), two mid-late flowering genotypes (Jangdae and Jinju) and eleven late flowering genotypes (Hongma300, Hongma74-3, Everglades41, ACC3748, ACC4111, ACC4153, ACC4139, ACC4751, ACC4985, ACC 5014 and ACC5072). Group 3 contained two genotypes (C19 and $\mathrm{C16}$ ) with same morphological phenotype. C13 genotype was not belonging to any groups.

Pearson's correlation coefficients based on average quantified values for morphology and phenolic compound data are shown in Table 4. KGRI, KRX and TPC contents were correlated with flowering date, corolla color and petal color. CAI content was correlated with flowering date. Plant height, $\mathrm{DM}$ yield and seed yield were correlated with flowering 
Table 3 Phenolic compound constituents in different kenaf genotypes

\begin{tabular}{|c|c|c|c|c|c|c|}
\hline Genotypes & $\mathrm{CAI}^{\mathrm{Z}}$ & $\mathrm{CA}^{\mathrm{y}}$ & $\mathrm{KGRI}^{\mathrm{x}}$ & $\mathrm{KRX}^{\mathrm{w}}$ & $\mathrm{KAPT}^{\mathrm{v}}$ & $\mathrm{TPC}^{\mathrm{u}}$ \\
\hline C9 & $27.0 \pm 4.8^{\mathrm{e}}$ & $69.8 \pm 0.2^{\mathrm{ij}}$ & $29.8 \pm 4.2^{\mathrm{h}}$ & $11.1 \pm 2.2^{\mathrm{gh}}$ & $97.8 \pm 1.7^{\mathrm{t}}$ & $317.0 \pm 18.8^{1}$ \\
\hline $\mathrm{C} 10$ & $27.7 \pm 5.0^{\mathrm{e}}$ & $90.8 \pm 6.9^{\mathrm{de}}$ & $45.1 \pm 5.6^{\mathrm{g}}$ & $15.8 \pm 1.7^{\mathrm{ef}}$ & $152.1 \pm 2.5^{1}$ & $451.6 \pm 28.1^{\mathrm{e}}$ \\
\hline C11 & $26.7 \pm 4.1^{\mathrm{e}}$ & $77.6 \pm 3.2^{\mathrm{gh}}$ & $41.1 \pm 5.6^{\mathrm{g}}$ & $14.5 \pm 2.2^{\mathrm{fg}}$ & $136.4 \pm 2.0^{\mathrm{p}}$ & $387.9 \pm 20.5^{\mathrm{i}}$ \\
\hline $\mathrm{C} 12$ & $33.5 \pm 5.0^{\mathrm{c}}$ & $96.3 \pm 3.8^{\mathrm{d}}$ & $69.8 \pm 9.6^{\mathrm{cd}}$ & $21.5 \pm 3.1^{\mathrm{c}}$ & $180.9 \pm 0.9^{\mathrm{i}}$ & $531.9 \pm 22.2^{\mathrm{d}}$ \\
\hline $\mathrm{C} 13$ & $30.7 \pm 4.9^{d}$ & $80.7 \pm 2.5^{\mathrm{fg}}$ & $48.9 \pm 6.7^{\mathrm{f}}$ & $16.5 \pm 2.5^{\mathrm{e}}$ & $140.6 \pm 1.8^{\circ}$ & $411.2 \pm 19.3^{\mathrm{h}}$ \\
\hline C14 & $26.2 \pm 4.3^{\mathrm{e}}$ & $76.8 \pm 2.9^{\mathrm{hi}}$ & $45.2 \pm 5.3^{\mathrm{g}}$ & $16.5 \pm 2.0^{\mathrm{e}}$ & $146.0 \pm 2.5^{\mathrm{m}}$ & $409.4 \pm 21.2^{\mathrm{h}}$ \\
\hline $\mathrm{C} 15$ & $23.2 \pm 3.4^{\mathrm{fg}}$ & $80.2 \pm 3.6^{\mathrm{fg}}$ & $47.0 \pm 6.5^{\mathrm{f}}$ & $15.7 \pm 3.6^{\mathrm{ef}}$ & $127.3 \pm 0.9^{q}$ & $387.7 \pm 13.5^{\mathrm{i}}$ \\
\hline $\mathrm{C} 16$ & $20.9 \pm 3.5^{\mathrm{g}}$ & $66.4 \pm 4.5^{\mathrm{Im}}$ & $33.7 \pm 4.5^{\mathrm{g}}$ & $10.6 \pm 0.5^{\mathrm{hi}}$ & $112.4 \pm 0.8^{r}$ & $332.0 \pm 16.3^{\mathrm{k}}$ \\
\hline $\mathrm{C} 17$ & $25.5 \pm 3.8^{\mathrm{e}}$ & $69.0 \pm 2.7^{\mathrm{ij}}$ & $35.3 \pm 4.9^{\mathrm{g}}$ & $12.8 \pm 2.8^{\mathrm{g}}$ & $100.6 \pm 0.1^{\mathrm{t}}$ & $330.3 \pm 13.0^{\mathrm{k}}$ \\
\hline C18 & $27.4 \pm 4.3^{\mathrm{e}}$ & $76.7 \pm 0.1^{\text {ih }}$ & $39.0 \pm 5.2^{\mathrm{g}}$ & $14.4 \pm 1.9^{\mathrm{fg}}$ & $123.8 \pm 1.3^{q}$ & $376.1 \pm 18.3^{\mathrm{j}}$ \\
\hline C19 & $31.4 \pm 5.3^{\mathrm{d}}$ & $78.8 \pm 1.6^{\mathrm{fg}}$ & $43.5 \pm 5.3^{\mathrm{g}}$ & $16.9 \pm 2.8^{\mathrm{ef}}$ & $150.8 \pm 1.2^{1}$ & $410.8 \pm 19.7^{\mathrm{h}}$ \\
\hline $\mathrm{C} 20$ & $26.9 \pm 4.1^{\mathrm{e}}$ & $60.4 \pm 2.0^{\mathrm{m}}$ & $32.7 \pm 4.4^{\mathrm{g}}$ & $11.9 \pm 2.1^{\mathrm{gh}}$ & $106.2 \pm 0.5^{\mathrm{s}}$ & $318.8 \pm 13.3^{1}$ \\
\hline $\mathrm{C} 22$ & $18.0 \pm 1.8^{\mathrm{g}}$ & $153.8 \pm 10.7^{\mathrm{a}}$ & $98.9 \pm 14.6^{\mathrm{ab}}$ & $33.2 \pm 3.1^{\mathrm{a}}$ & $306.1 \pm 1.1^{\mathrm{a}}$ & $766.8 \pm 47.9^{\mathrm{a}}$ \\
\hline C14RS1 & $30.8 \pm 4.0^{\mathrm{d}}$ & $74.1 \pm 0.5^{\mathrm{i}}$ & $37.0 \pm 5.9^{\mathrm{g}}$ & $10.0 \pm 0.1^{\mathrm{hi}}$ & $98.7 \pm 3.0^{t}$ & $364.8 \pm 16.8^{\mathrm{j}}$ \\
\hline C14RS2 & $31.5 \pm 4.9^{\mathrm{d}}$ & $73.4 \pm 1.3^{\mathrm{i}}$ & $36.9 \pm 5.4^{\mathrm{g}}$ & $13.1 \pm 2.4^{\mathrm{fg}}$ & $113.4 \pm 0.6^{\mathrm{r}}$ & $280.4 \pm 8.3^{\mathrm{m}}$ \\
\hline Jeokbong & $7.4 \pm 0.3^{\mathrm{h}}$ & $19.0 \pm 0.8^{\mathrm{n}}$ & $43.3 \pm 5.7^{\mathrm{g}}$ & $10.9 \pm 2.5^{\mathrm{hi}}$ & $139.1 \pm 5.3^{\circ}$ & $371.8 \pm 9.5^{\mathrm{j}}$ \\
\hline Baekma & $24.0 \pm 1.6^{\mathrm{ef}}$ & $67.2 \pm 9.7^{\mathrm{kl}}$ & $33.4 \pm 5.8^{\mathrm{g}}$ & $9.1 \pm 0.0^{\mathrm{i}}$ & $146.2 \pm 2.9^{\mathrm{m}}$ & $364.2 \pm 1.0^{\mathrm{j}}$ \\
\hline WFM1-2 & $22.0 \pm 1.5^{\mathrm{f}}$ & $64.9 \pm 12.1^{\mathrm{lm}}$ & $32.3 \pm 6.9^{g}$ & $8.7 \pm 0.3^{\mathrm{i}}$ & $194.6 \pm 2.6^{\mathrm{h}}$ & $441.3 \pm 4.8^{\mathrm{f}}$ \\
\hline Jangdae & $26.6 \pm 3.7^{\mathrm{e}}$ & $88.3 \pm 8.8^{\text {de }}$ & $75.7 \pm 9.6^{c}$ & $16.3 \pm 2.3^{\mathrm{ef}}$ & $145.1 \pm 4.0^{\mathrm{m}}$ & $504.5 \pm 11.4^{\mathrm{d}}$ \\
\hline Jinju & $32.8 \pm 4.5^{\mathrm{c}}$ & $91.5 \pm 1.1^{\mathrm{de}}$ & $86.3 \pm 10.3^{\mathrm{b}}$ & $21.5 \pm 4.5^{\mathrm{c}}$ & $191.0 \pm 2.8^{\mathrm{h}}$ & $546.5 \pm 11.1^{\mathrm{d}}$ \\
\hline Hongma300 & $26.4 \pm 3.5^{\mathrm{e}}$ & $88.8 \pm 7.9^{\mathrm{d}}$ & $75.9 \pm 9.2^{c}$ & $16.3 \pm 2.2^{\mathrm{ef}}$ & $146.6 \pm 2.4^{\mathrm{m}}$ & $444.2 \pm 8.5^{\mathrm{f}}$ \\
\hline Hongma74-3 & $25.5 \pm 3.2^{\mathrm{e}}$ & $94.3 \pm 5.6^{\mathrm{d}}$ & $79.2 \pm 10.2^{\mathrm{c}}$ & $18.9 \pm 2.1^{\mathrm{de}}$ & $174.7 \pm 3.4^{\mathrm{j}}$ & $492.3 \pm 8.8^{\mathrm{d}}$ \\
\hline Everglades41 & $25.5 \pm 3.7^{\mathrm{e}}$ & $83.2 \pm 2.8^{\text {efg }}$ & $75.9 \pm 11.2^{\mathrm{c}}$ & $19.0 \pm 2.1^{\text {de }}$ & $182.1 \pm 2.3^{\mathrm{i}}$ & $729.5 \pm 8.1^{\mathrm{a}}$ \\
\hline ACC3748 & $41.5 \pm 7.3^{\mathrm{bc}}$ & $113.7 \pm 0.6^{\mathrm{c}}$ & $107.5 \pm 12.7^{\mathrm{a}}$ & $26.0 \pm 3.7^{\mathrm{bc}}$ & $241.4 \pm 0.5^{\mathrm{d}}$ & $672.2 \pm 25.7^{b}$ \\
\hline ACC4111 & $44.7 \pm 6.7^{\mathrm{b}}$ & $113.2 \pm 9.4^{\mathrm{c}}$ & $108.6 \pm 16.2^{\mathrm{a}}$ & $30.8 \pm 3.6^{\mathrm{ab}}$ & $263.9 \pm 2.8^{\mathrm{b}}$ & $694.8 \pm 15.6^{\mathrm{a}}$ \\
\hline ACC4153 & $39.2 \pm 6.4^{\mathrm{c}}$ & $91.2 \pm 2.3^{\mathrm{de}}$ & $63.4 \pm 8.8^{\mathrm{de}}$ & $19.5 \pm 3.6^{\mathrm{de}}$ & $217.5 \pm 0.0^{f}$ & $517.4 \pm 7.3^{\mathrm{d}}$ \\
\hline ACC4139 & $36.7 \pm 5.8^{\mathrm{c}}$ & $96.2 \pm 3.3^{\mathrm{d}}$ & $86.2 \pm 11.4^{\mathrm{c}}$ & $22.7 \pm 2.7^{\mathrm{c}}$ & $164.2 \pm 0.0^{\mathrm{k}}$ & $571.5 \pm 19.2^{\mathrm{c}}$ \\
\hline ACC4443 & $20.0 \pm 2.5^{\mathrm{fg}}$ & $82.1 \pm 2.7^{\mathrm{efg}}$ & $70.1 \pm 9.8^{\mathrm{cd}}$ & $18.9 \pm 2.4^{\mathrm{de}}$ & $165.7 \pm 0.5^{\mathrm{k}}$ & $482.5 \pm 1.8^{\mathrm{d}}$ \\
\hline ACC4751 & $28.1 \pm 4.4^{\mathrm{de}}$ & $71.5 \pm 2.6^{\mathrm{ij}}$ & $86.9 \pm 12.7^{\mathrm{b}}$ & $27.4 \pm 3.3^{\mathrm{b}}$ & $229.0 \pm 0.5^{\mathrm{e}}$ & $571.0 \pm 3.6^{\mathrm{c}}$ \\
\hline ACC4985 & $26.1 \pm 3.8^{\mathrm{e}}$ & $68.4 \pm 1.0^{\mathrm{jk}}$ & $61.7 \pm 7.5^{\mathrm{de}}$ & $15.9 \pm 3.3^{\mathrm{ef}}$ & $142.1 \pm 0.3^{\mathrm{n}}$ & $421.0 \pm 1.1^{\mathrm{g}}$ \\
\hline ACC5014 & $40.3 \pm 5.3^{\mathrm{bc}}$ & $114.0 \pm 13.0^{\mathrm{c}}$ & $89.1 \pm 14.4^{\mathrm{b}}$ & $30.6 \pm 4.9^{\mathrm{ab}}$ & $246.7 \pm 4.9^{c}$ & $647.2 \pm 8.2^{\mathrm{b}}$ \\
\hline ACC5072 & $64.0 \pm 10.9^{\mathrm{a}}$ & $130.4 \pm 2.6^{\mathrm{b}}$ & $68.3 \pm 9.9^{\mathrm{d}}$ & $30.5 \pm 8.1^{\mathrm{ab}}$ & $202.8 \pm 4.0^{\mathrm{g}}$ & $707.1 \pm 17.5^{\mathrm{a}}$ \\
\hline
\end{tabular}

${ }^{\mathrm{z}}$ CAI: Chlorogenic acid isomer, ${ }^{\mathrm{y}} \mathrm{CA}$ : Chlorogenic acid, ${ }^{\mathrm{x}} \mathrm{KGRI}$ : Kaempferol glucosyl rhamnoside isomer, ${ }^{\mathrm{w}} \mathrm{KRX}$ : Kaempferol rhamnosyl xyloside, "KAPT: Kaemperitrin, The letters above each point indicate a significant difference at the $5 \%$ level (Duncan's multiple range tests, $n=3$ ).

date, KGRI, KRX, KAPT.

Genetic diversity and relationships

The marker attributes for the SSR primers were summarized as number of allele (NA), number of polymorphic allele (NPA), fraction of polymorphic markers (FPM), GD, PIC, EMR, and MI (Table 5). A total of 229 SSR allele were detected overall, and there were 217 polymorphic allele (94.8\%) among the cultivars. The NA for each of the SSR primers ranged from 2 to 8 , with a mean of 2.90. The NPA of the SSR primers ranged from 2 to 8 , with a mean of 2.75 . The average FPM was 0.94 per marker, and the range was from 0.5 to 1 . The GD ranged from 0.09 to 0.83 , with an average of 0.44 , and the PIC values ranged from 0.09 to 0.81 , with a mean value of 0.37 . The maximum values of
GD and PIC occurred with the KU896464, whereas the minimum values were generated by the DQ068364. The SI values of the SSR primers ranged from 0.02 to 0.73 , with a mean of 0.45 . The maximum values of SI occurred with KU896449 (Table 5).

A phylogenetic tree was constructed based on the 80 SSR markers in 32 kenaf genotypes (Fig. 2C). The genotypes divided into early flowering, mid-late flowering, and non-flowering groups were grouped into four distinct groups with the exceptions of the two (ACC3748 and ACC5072) genotypes. Group 1 consisted of eighteen early flowering genotypes. Group 2 included both of the non-flowering genotypes (ACC4443 and ACC4751). Group 3 contained eight non-flowering genotypes (Hongma300, Hongma74-3, ACC5014, Everglades41, ACC4985, ACC4135, ACC4111 and ACC4139). Group 4 included mid-late mutant cultivar (Jangdae) and these of original 

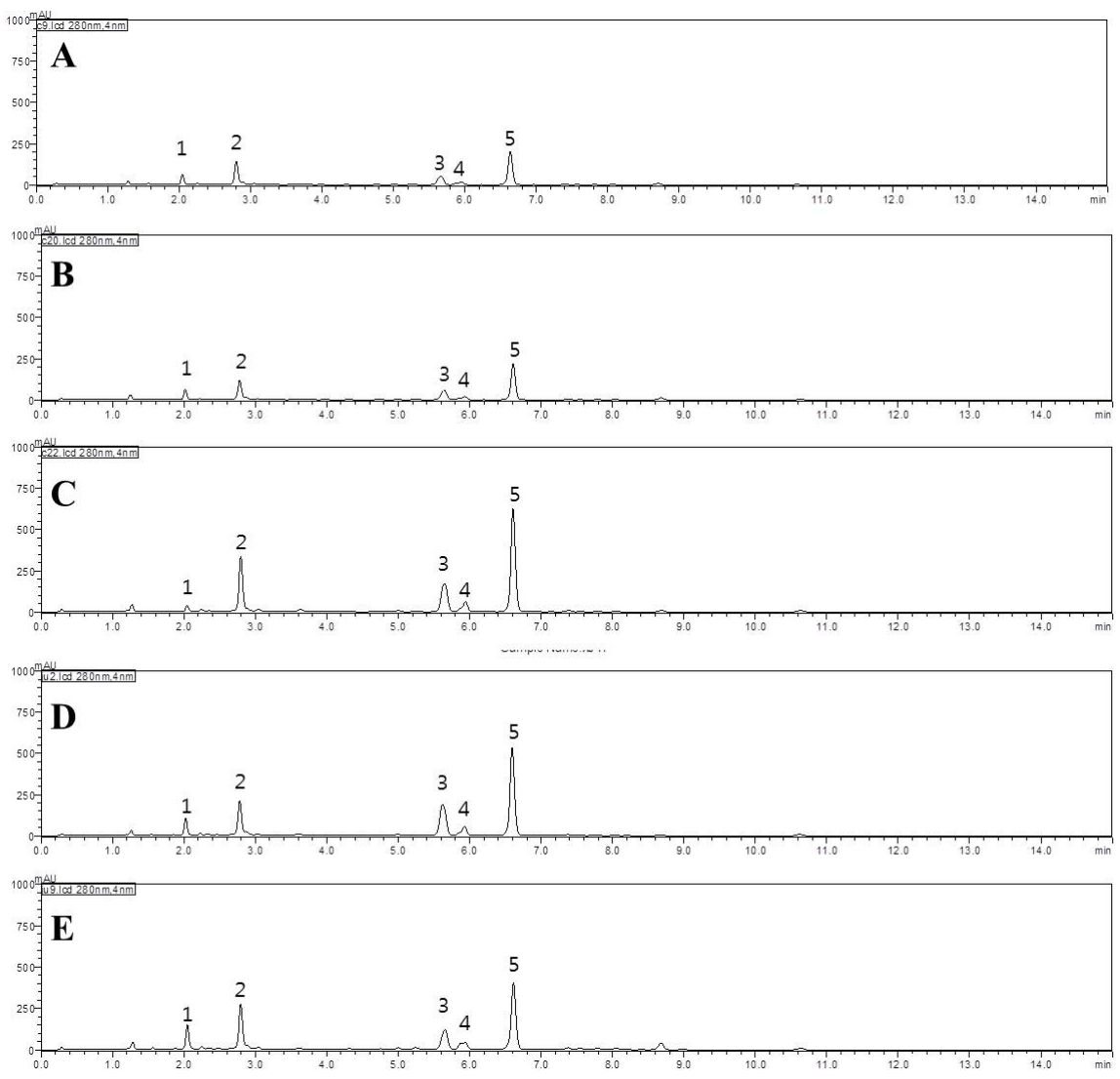

Fig. 3 Ultra-high performance liquid chromatography (UPLC) chromatogram of kenaf phenolic compounds detected at $280 \mathrm{~nm}$. Peaks $1,2,3,4$, and 5 are chlorogenic acid isomer (CAI), chlorogenic acid (CA), kaempferol glucosyl rhamnoside isomer (KGRI), kaempferol rhamnosyl xyloside (KRX), and kaemperitrin (KAPT), respectively. A: C9, B: C20, C: C22, D: ACC4111, and E: ACC5072

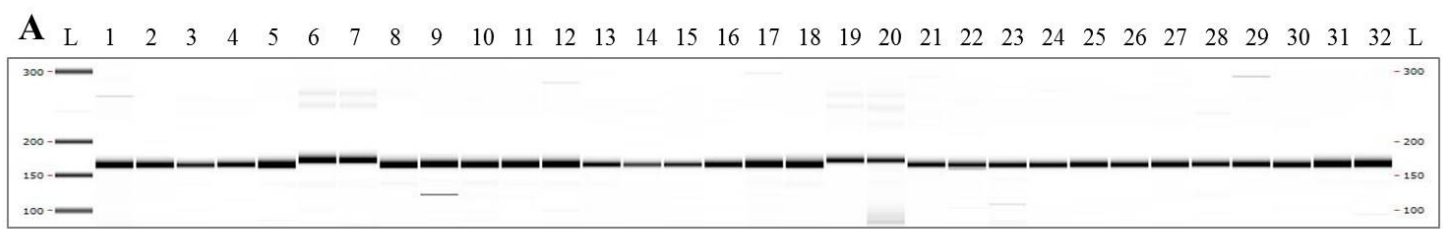

\section{B}

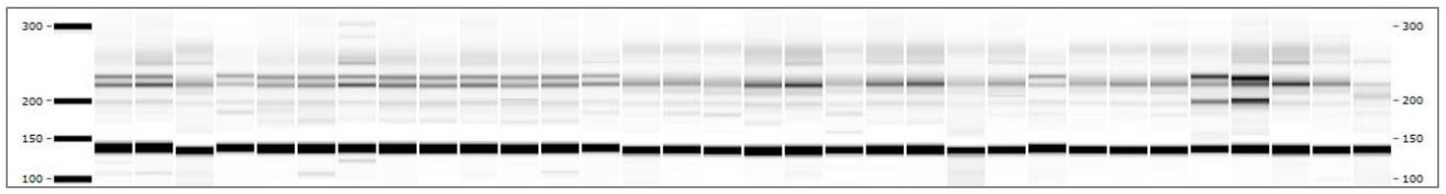

C

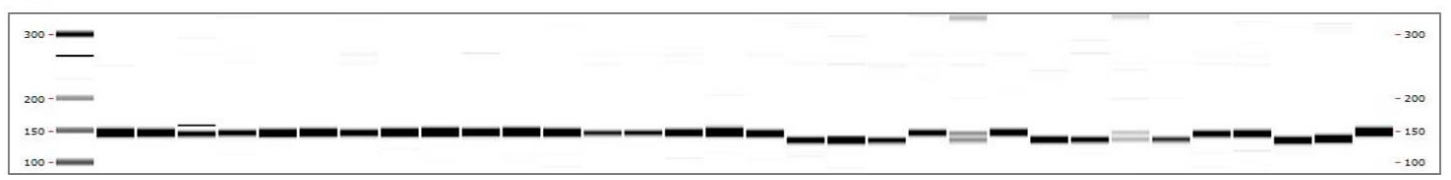

Fig. 4 Caliper LabChip GX II patterns following PCR amplification for polymorphic SSR markers in kenaf genotypes. A: KU896464, B: KU896435, C: KU896449. Primer sequences are listed in Table 5

genotype (Jinju).

We explored correlation analysis among the SSR markers, chemical components and morphological characteristics of the kenaf cultivars by applying Q GLM and Q + K MLM statistical methods (Table 6). A total of 15 significant correlations $(p<0.05)$ were revealed using the Q GLM and $\mathrm{Q}+\mathrm{K}$ MLM statistical approaches. We discovered four (KU896387, KU896399, KU896441 and KU896449) significant 
Table 4 Correlation coefficients between flavonoid content, yield and morphological characteristics of kenaf genotypes

\begin{tabular}{|c|c|}
\hline Trait & $\mathrm{R}^{2}$ \\
\hline $\mathrm{CAI}^{\mathrm{z}}$ content and flowering date & 0.460 \\
\hline $\mathrm{CA}^{\mathrm{y}}$ content and leaf color & 0.507 \\
\hline $\mathrm{KGRI}^{\mathrm{x}}$ content and flowering date & 0.750 \\
\hline $\mathrm{KRX}^{\mathrm{w}}$ content and flowering date & 0.597 \\
\hline $\mathrm{KAPT}^{\mathrm{u}}$ content and flowering date & 0.553 \\
\hline $\mathrm{TPC}^{\mathrm{t}}$ content and flowering date & 0.638 \\
\hline KGRI and corolla color & 0.611 \\
\hline KRX and corolla color & 0.496 \\
\hline KAPT and corolla color & 0.462 \\
\hline TPC and corolla color & 0.516 \\
\hline KGRI and petal color & 0.616 \\
\hline KRX and petal color & 0.499 \\
\hline KAPT and petal color & 0.521 \\
\hline TPC and petal color & 0.576 \\
\hline Plant height and flowering date & 0.940 \\
\hline $\mathrm{DM}^{\mathrm{s}}$ yield and flowering date & 0.985 \\
\hline Seed yield and flowering date & 0.901 \\
\hline KGRI and Plant height & 0.697 \\
\hline KGRI and DM yield & 0.741 \\
\hline KGRI and Seed yield & 0.705 \\
\hline KRX and Plant height & 0.456 \\
\hline KRX and DM yield & 0.557 \\
\hline KRX and Seed yield & 0.644 \\
\hline KAPT and Plant height & 0.469 \\
\hline KAPT and DM yield & 0.526 \\
\hline KAPT and Seed yield & 0.597 \\
\hline
\end{tabular}

${ }^{\mathrm{z}} \mathrm{CAI}$ : Chlorogenic acid isomer, ${ }^{\mathrm{y}} \mathrm{CA}$ : Chlorogenic acid, ${ }^{\mathrm{x}} \mathrm{KGRI}$ : Kaempferol glucosyl rhamnoside isomer, "KRX: Kaempferol rhamnosyl xyloside, "KAPT: Kaemperitrin, ${ }^{\mathrm{t}} \mathrm{TPC}$ : Total phenolic content, ${ }^{\text {sDM: Dry matter }}$

correlated with leaf color. The lowest $p$-value was observed for the KU896449 with leaf color $\left(P=2.34 \mathrm{E}-204, \mathrm{R}^{2}=\right.$ 0.974) in Q GLM. Three SSR markers (KU896384, KU896438 and KU896440) had highly significant correlated with petal color. The KU896440 marker had the lowest $p$-value $(P=$ $\left.1.36 \mathrm{E}-07, \mathrm{R}^{2}=0.206\right)$ by Q MLM. The KU896377 and KU896423 markers had significant correlated with vein color. The KU896426 marker had significant correlated $(P=$ $0.004, \mathrm{R}^{2}=0.159$ ) with KGRI contents. The KU896408 and KU896449 markers had significant correlated with stem color. KU896408 had the lowest $p$-value $\left(P=0.001, \mathrm{R}^{2}=\right.$ $0.166)$ by Q GLM. KU896449 markers had significant (Q GLM: $P=0.002, \mathrm{R} 2=0.367$ and Q+K MLM: $P=0.023$, $\left.\mathrm{R}^{2}=0.289\right)$ correlated with corolla color.

\section{Discussion}

Kenaf is promising potential to exploit and utilize, but identification of kenaf genotypes and understanding of morphological, chemical and genetic characteristics and relationships is limited, thus significantly hinders their effective utilization (Alexopoulou et al. 2013; Faruq et al. 2013; Jeong et al. 2017). Genetic analyses of the complex quantitative traits involved in morphology and functional compounds are limited (Alexopoulou et al. 2013; Faruq et al. 2013; Zhang et al. 2015). This preliminary study aimed to understand the morphological, chemical and genetic characteristics of these economically important traits, and it is the first exploration of the morphological, chemical and genetic diversity and relationship of kenaf genotype.

Kenaf is considered an important medicinal crop in India and South Africa (Alexopoulou et al. 2013). The leaf has been reported to be anodyne, aperitif, aphrodisiacal, fattening, purgative, and stomachic and has long been used as a traditional medicine in Africa and India (Kubmarawa et al., 2009; Alexopoulou et al. 2013; Jin et al., 2013). The leaf contains large amounts of polyphenols, tannins, and other mineral compounds (Kobaisy et al., 2001; Ryu et al., 2017a). Most of the medicinal benefits attributed to kenaf are due to the presence of phenolic compounds (Jin et al. 2013; Zhao et al. 2014; Ryu et al. 2017). The major bioactive compounds in kenaf include the CAI, CA, KGRI, KRX and KAPT. In this study, their content and TPC had a high correlation with flowering date, hypocotyl color corolla color and petal color. KAPT is the main compound present in kenaf leaf. The compound has an acute lowering effect on the blood glucose level of diabetic rats (Jorge et al., 2004). In other studies, KAPT has shown high reactivity with 1,1-diphenyl-2-picrylhydrazyl (DPPH) free radicals, angiotensin I converting enzyme (ACE) inhibition and also decreased lipid peroxidation (Cazarolli et al. 2006; Jin et al., 2013). The highest level of KAPT content was observed in the C22 genotypes (306.1 mg/100 g) and the lowest level was observed in the C9 genotypes $(97.8 \mathrm{mg} / 100 \mathrm{~g})$. Ryu et al. (2017) reported that the KAPT content ranged from 10 to $178 \mathrm{mg} / 100 \mathrm{~g}$ with different solvent extract of kenaf leaves, and that water has the highest KAPT content. The high levels of these compounds observed in the present study, especially for the $\mathrm{C} 22$ genotypes, indicate promising commercial potential for kenaf as a source of phenolic compounds.

Analyses of genetic diversity provide for the selection of genotypes in breeding programs and provide useful genetic information (Zhang et al. 2015; Jeong et al., 2017). In this study, high levels of genetic diversity were found for the various kenaf genotypes. PIC and GD detected in this study for kenaf are higher than those previously observed using other marker (SRAP, ISSR, RAPD and AFLP) systems 
Table 5 Characteristics of SSR markers for the identification of kenaf genotypes

\begin{tabular}{|c|c|c|c|c|c|c|c|c|}
\hline Genbank ID & Primer sequences $\left(5^{\prime}-3^{\prime}\right)$ & Origin & $\mathrm{NA}^{\mathrm{z}}$ & $\mathrm{NPA}^{\mathrm{y}}$ & $\mathrm{FPM}^{\mathrm{x}}$ & $\mathrm{GD}^{\mathrm{w}}$ & $\mathrm{PIC}^{\mathrm{V}}$ & $\mathrm{SI}^{\mathrm{u}}$ \\
\hline DQ068360 & $\begin{array}{l}\text { F CTAGTTTTTGCAGAGGCCAAGT } \\
\text { R AGAAGAATTGTTGGCCATGTCT }\end{array}$ & & 3 & 3 & 1.00 & 0.51 & 0.46 & 0.46 \\
\hline DQ068361 & $\begin{array}{l}\text { F ACTAGTTTTTGCAGAGGCCAAG } \\
\text { R GATTACTCATTTGGCCATGTCTC }\end{array}$ & & 5 & 5 & 1.00 & 0.77 & 0.68 & 0.73 \\
\hline DQ068362 & $\begin{array}{l}\text { F TTACTACCGTTTGAGCGGAGA } \\
\text { R CGAATGCCAAGAAAGTTTCAG }\end{array}$ & & 3 & 2 & 0.67 & 0.36 & 0.22 & 0.33 \\
\hline DQ068364 & $\begin{array}{l}\text { F GAGGCACTTCAGTGTCGTAGC } \\
\text { R CCAATAGGCAGGTTTTTCCTC }\end{array}$ & & 2 & 1 & 0.50 & 0.09 & 0.09 & 0.02 \\
\hline DQ068366 & $\begin{array}{l}\text { F } \text { TGCCCATTTTTGAGTTTTCAC } \\
\text { R TCCTCGAGAAAAAGGATTGTG }\end{array}$ & $\begin{array}{l}\text { Genomic SSR } \\
\text { Markers }\end{array}$ & 2 & 1 & 0.50 & 0.12 & 0.11 & 0.12 \\
\hline DQ068373 & $\begin{array}{l}\text { F ACTAGTTTTTGCAGAGGCCAAG } \\
\text { R GAGTGTTGTGCATGAAAGGAAA }\end{array}$ & & 3 & 2 & 0.67 & 0.54 & 0.48 & 0.42 \\
\hline DQ068374 & $\begin{array}{l}\text { F CACTCCAATCACCATTCACG } \\
\text { R CTGATCGAATCCAACCCCTA }\end{array}$ & & 2 & 1 & 0.50 & 0.09 & 0.09 & 0.02 \\
\hline DQ068376 & $\begin{array}{l}\text { F CAGTAGCGGACCGTTATTTGA } \\
\text { R TTACAGCCTTGGGACTTCAGA }\end{array}$ & & 3 & 3 & 1.00 & 0.49 & 0.43 & 0.24 \\
\hline DQ068377 & $\begin{array}{l}\text { F GAATGCAACATTTTTAAATGCAA } \\
\text { R GTCTACAAAAGCCAAAGCATACC }\end{array}$ & & 3 & 3 & 0.50 & 0.47 & 0.42 & 0.38 \\
\hline KU896377 & $\begin{array}{l}\text { F CCGAAGCTCCTGCTTTTATC } \\
\text { R GTCTCAGATGAAGCCACCAC }\end{array}$ & & 3 & 3 & 1.00 & 0.56 & 0.50 & 0.47 \\
\hline KU896380 & $\begin{array}{l}\text { F GAAGAAACGGGTCATTCCTC } \\
\text { R GTAGTCGTAGTCATCCTCTGCTC }\end{array}$ & & 3 & 2 & 0.67 & 0.53 & 0.42 & 0.65 \\
\hline KU896381 & $\begin{array}{l}\text { F GTGATATCCGAGCACCTTTG } \\
\text { R GCGATGATATCAGAACCTCGTC }\end{array}$ & & 2 & 2 & 1.00 & 0.50 & 0.37 & 0.58 \\
\hline KU896382 & $\begin{array}{l}\text { F GGTTTTCGGCTCTTGGTT } \\
\text { R CTGGACAATTGCGAGAAGAG }\end{array}$ & & 3 & 3 & 1.00 & 0.36 & 0.29 & 0.48 \\
\hline KU896383 & $\begin{array}{l}\text { F GCTGAGCAGAGGAGTAGAAGAA } \\
\text { R TTCAGCTCAAGCAGTATCCC }\end{array}$ & & 2 & 2 & 1.00 & 0.58 & 0.49 & 0.60 \\
\hline KU896384 & $\begin{array}{l}\text { F ATCCTAGTGGATCCCTGAACTC } \\
\text { R GACGATGAGGAGCAGAAAGA }\end{array}$ & & 2 & 2 & 1.00 & 0.43 & 0.34 & 0.66 \\
\hline KU896385 & $\begin{array}{l}\text { F GCTGCCATGCTCATGATT } \\
\text { R AGCTCACCCTCCACTTCTCTAT }\end{array}$ & & 2 & 2 & 1.00 & 0.48 & 0.37 & 0.63 \\
\hline KU896386 & $\begin{array}{l}\text { F ACAGCTTTGACTGTCGTCACTG } \\
\text { R AAGTATCTTGTGGGCTGTGG }\end{array}$ & & 2 & 2 & 1.00 & 0.45 & 0.35 & 0.41 \\
\hline KU896387 & $\begin{array}{l}\text { F GCCTTCGGAGTAAATGGGT } \\
\text { R CACCCAAACATTCTCTCTGG }\end{array}$ & EST-SSR & 7 & 7 & 1.00 & 0.32 & 0.27 & 0.27 \\
\hline KU896388 & $\begin{array}{l}\text { F GTTGGTCGTAAAAGCCGAG } \\
\text { R AACCCCGTCTTTAACCTCAG }\end{array}$ & Markers & 2 & 2 & 1.00 & 0.74 & 0.72 & 0.35 \\
\hline KU896389 & $\begin{array}{l}\text { F GAAACGAAGGGTAGAGTACGGT } \\
\text { R GCAGTGTAAACAAACAGCCC }\end{array}$ & & 3 & 3 & 1.00 & 0.17 & 0.16 & 0.44 \\
\hline KU896390 & $\begin{array}{l}\text { F AGATTGATCTCGTCACCCCT } \\
\text { R CCAAACTGGATCGTAATCCG }\end{array}$ & & 2 & 2 & 1.00 & 0.49 & 0.38 & 0.55 \\
\hline KU896391 & $\begin{array}{l}\text { F CAGAAAAGTAGCGGGATGAG } \\
\text { R CCACTCGACATTAAACCCAC }\end{array}$ & & 2 & 2 & 1.00 & 0.41 & 0.33 & 0.54 \\
\hline KU896395 & $\begin{array}{l}\text { F TACTGGATGAAGGAGTAGCAGC } \\
\text { R CTTGATAGGCATCCCTTACCC }\end{array}$ & & 2 & 2 & 1.00 & 0.38 & 0.31 & 0.36 \\
\hline KU896397 & $\begin{array}{l}\text { F CCATATAGTTTGGGGGAAGG } \\
\text { R CAGTGAGAAGTGAGTGGCTACA }\end{array}$ & & 2 & 2 & 1.00 & 0.19 & 0.18 & 0.61 \\
\hline KU896399 & $\begin{array}{l}\text { F AGCCTGTGCTGAAAGCTAGA } \\
\text { R GGAGGGAGCATAAGTGAGTTTG }\end{array}$ & & 3 & 3 & 1.00 & 0.46 & 0.35 & 0.38 \\
\hline KU896400 & $\begin{array}{l}\text { F CCGACAAGAACAAGTCCA } \\
\text { R CAACCCGTGTGCATTGAG }\end{array}$ & & 3 & 3 & 1.00 & 0.42 & 0.35 & 0.41 \\
\hline KU896404 & $\begin{array}{l}\text { F GATGGTTTCTCCCAACAACC } \\
\text { R CAACGACATCGTCGTCTTC }\end{array}$ & & 2 & 2 & 1.00 & 0.52 & 0.41 & 0.62 \\
\hline
\end{tabular}


Table 5 Continued

\begin{tabular}{|c|c|c|c|c|c|c|c|c|}
\hline Genbank ID & Primer sequences $\left(5^{\prime}-3^{\prime}\right)$ & Origin & $\mathrm{NA}^{\mathrm{z}}$ & $\mathrm{NPA}^{\mathrm{y}}$ & FPM $^{\mathrm{x}}$ & $\mathrm{GD}^{\mathrm{w}}$ & $\mathrm{PIC}^{\mathrm{v}}$ & $\mathrm{SI}^{\mathrm{u}}$ \\
\hline KU896405 & $\begin{array}{l}\text { F GTCGTCATCATCGTCCAATC } \\
\text { R AGATCTCTCTTCACAGTGTCCC }\end{array}$ & & 4 & 4 & 1.00 & 0.47 & 0.36 & 0.39 \\
\hline KU896406 & $\begin{array}{l}\text { F CAGTCTGCATCGTCCAATC } \\
\text { R AGATCTCTCTTCACAGTGTCCC }\end{array}$ & & 3 & 3 & 1.00 & 0.66 & 0.61 & 0.33 \\
\hline KU896407 & $\begin{array}{l}\text { F GCCTTCAGAGAATAGATGTGGG } \\
\text { R CAGTTCATCGACTTGGCTTG }\end{array}$ & & 3 & 2 & 0.67 & 0.33 & 0.30 & 0.49 \\
\hline KU896408 & $\begin{array}{l}\text { F CTAACACGTCCGGCAACA } \\
\text { R GGAGTTCAAGAGGACGTAGTTG }\end{array}$ & & 3 & 2 & 0.67 & 0.22 & 0.19 & 0.62 \\
\hline KU896409 & $\begin{array}{l}\text { F CCTCAAGCTCCTCGTAATACAC } \\
\text { R GGGTACCAGTGAAGAGAACAAG }\end{array}$ & & 2 & 2 & 1.00 & 0.47 & 0.36 & 0.49 \\
\hline KU896410 & $\begin{array}{l}\text { F GTACTTGACGTAGGAAAGGCAG } \\
\text { R TTATACGACTCCCCACGGA }\end{array}$ & & 3 & 3 & 1.00 & 0.22 & 0.19 & 0.42 \\
\hline KU896411 & $\begin{array}{l}\text { F GTTCCTATGAAGAATCCGGC } \\
\text { R ACTTTGAGAGGTTGCAAGGG }\end{array}$ & & 2 & 2 & 1.00 & 0.52 & 0.45 & 0.56 \\
\hline KU896412 & $\begin{array}{l}\text { F GTAATCGTTGTTGGCGTTGG } \\
\text { R GTCAAACACAAGCTCCAGTCC }\end{array}$ & & 7 & 7 & 1.00 & 0.40 & 0.32 & 0.31 \\
\hline KU896417 & $\begin{array}{l}\text { F CCCTCTACCTCTAGGATGATTCTC } \\
\text { R ACTAGGTTTCTCTTCAGCGGC }\end{array}$ & & 2 & 2 & 1.00 & 0.79 & 0.76 & 0.43 \\
\hline KU896418 & $\begin{array}{l}\text { F GTTCCTTGAGAGAAAGGAGAGG } \\
\text { R GTGTTAGTGAGGAGAAGCAAGG }\end{array}$ & & 3 & 3 & 1.00 & 0.20 & 0.18 & 0.55 \\
\hline KU896419 & $\begin{array}{l}\text { F GGTAAACTGTTGAAGCGGGT } \\
\text { R GCAGAGCATTTCAACCAG }\end{array}$ & & 2 & 2 & 1.00 & 0.65 & 0.58 & 0.42 \\
\hline KU896420 & $\begin{array}{l}\text { F cCCCTTTTGATCTCTTGC } \\
\text { R AGGAGGGAGAGAGAGCTTCA }\end{array}$ & & 2 & 2 & 1.00 & 0.26 & 0.23 & 0.52 \\
\hline KU896422 & $\begin{array}{l}\text { F GGCTGCCCTTGCTAATAAGT } \\
\text { R ACTCGCTTCTTCATGCTCC }\end{array}$ & & 2 & 2 & 1.00 & 0.38 & 0.30 & 0.59 \\
\hline KU896423 & $\begin{array}{l}\text { F GGTGGTTTAAACGAGCACC } \\
\text { R GTCTCCCCATTGTTCCTGA }\end{array}$ & & 3 & 3 & 1.00 & 0.43 & 0.34 & 0.27 \\
\hline KU896424 & $\begin{array}{l}\text { F CATCCCGTCTAACACTACATCC } \\
\text { R GCACCGAGTATATCCTTCCAC }\end{array}$ & & 2 & 2 & 1.00 & 0.17 & 0.17 & 0.66 \\
\hline KU896426 & $\begin{array}{l}\text { F GGAGTCGTATAATGGGGTGA } \\
\text { R CTCCCTCTCGAAAATACGTAGC }\end{array}$ & & 3 & 3 & 1.00 & 0.50 & 0.37 & 0.44 \\
\hline KU896427 & $\begin{array}{l}\text { F GGTATGGCAGACGAGATGTT } \\
\text { R GTGTTAGTAGGCACTGGTGAAG }\end{array}$ & & 2 & 2 & 1.00 & 0.44 & 0.40 & 0.39 \\
\hline KU896428 & $\begin{array}{l}\text { F GCTCCTGCACTGTTTGTTGT } \\
\text { R CTAGGCTTATGTGTGGACCG }\end{array}$ & & 2 & 2 & 1.00 & 0.22 & 0.19 & 0.45 \\
\hline KU896429 & $\begin{array}{l}\text { F GGAGTGTCTTGTAATAGCCCAC } \\
\text { R CTCCAACCTCCCATTGTTC }\end{array}$ & & 4 & 4 & 1.00 & 0.30 & 0.26 & 0.39 \\
\hline KU896430 & $\begin{array}{l}\text { F GATCCGAAGGTAAATGGGTC } \\
\text { R CAGACACCTTTAGCCCCAC }\end{array}$ & & 2 & 2 & 1.00 & 0.64 & 0.60 & 0.40 \\
\hline KU896431 & $\begin{array}{l}\text { F AATCCAGGGAAGCAGCTC } \\
\text { R GCATATCTCTGAAGTGTCTCCG }\end{array}$ & & 4 & 4 & 1.00 & 0.22 & 0.20 & 0.39 \\
\hline KU896434 & $\begin{array}{l}\text { F CACTAAGAGCCCAGAAAGAAGC } \\
\text { R GAGACTCTTGTGGAGTTTCTGC }\end{array}$ & & 2 & 2 & 1.00 & 0.61 & 0.55 & 0.27 \\
\hline KU896435 & $\begin{array}{l}\text { F GTAGTCACCGCCGTCACAATAG } \\
\text { R CTATTCTGGCTCTCCCAACA }\end{array}$ & & 2 & 2 & 1.00 & 0.12 & 0.11 & 0.44 \\
\hline KU896436 & $\begin{array}{l}\text { F CGGCTGTTACTCCATCAAAG } \\
\text { R GGTCGTCTTACAATGGTTCC }\end{array}$ & & 3 & 3 & 1.00 & 0.22 & 0.19 & 0.44 \\
\hline KU896438 & $\begin{array}{l}\text { F ATTCAGAAACCGATGCCC } \\
\text { R GGAATGTCACTGGTCCGAG }\end{array}$ & & 3 & 3 & 1.00 & 0.49 & 0.41 & 0.59 \\
\hline KU896439 & $\begin{array}{l}\text { F AGCTCCGGGGATAAGTTAAG } \\
\text { R GCCTTCTCTCCTTGACCAGTA }\end{array}$ & & 3 & 3 & 1.00 & 0.61 & 0.53 & 0.42 \\
\hline KU896440 & $\begin{array}{l}\text { F GGCACAATAGAAGAGGCAGACT } \\
\text { R CTTCGAATTTCAGCGTCG }\end{array}$ & & 3 & 3 & 1.00 & 0.48 & 0.39 & 0.43 \\
\hline
\end{tabular}


Table 5 Continued

\begin{tabular}{|c|c|c|c|c|c|c|c|c|}
\hline Genbank ID & Primer sequences $\left(5^{\prime}-3^{\prime}\right)$ & Origin & $\mathrm{NA}^{\mathrm{z}}$ & $\mathrm{NPA}^{\mathrm{y}}$ & $\mathrm{FPM}^{\mathrm{x}}$ & $\mathrm{GD}^{\mathrm{w}}$ & $\mathrm{PIC}^{\mathrm{v}}$ & $\mathrm{SI}^{\mathrm{u}}$ \\
\hline KU896441 & $\begin{array}{l}\text { F GAGATGTTTGATGCTCCAGG } \\
\text { R TCACGAAACCAAAGCAGC }\end{array}$ & & 2 & 2 & 1.00 & 0.39 & 0.33 & 0.46 \\
\hline KU896442 & $\begin{array}{l}\text { F CACAGTTTCACGAGGCTAACTC } \\
\text { R GAGAAGAGCTTCCAACCAGG }\end{array}$ & & 8 & 8 & 1.00 & 0.28 & 0.24 & 0.22 \\
\hline KU896443 & $\begin{array}{l}\text { F GTACACAAAGTGCAACCTCTCC } \\
\text { R TTCTCCCCTAATTCCTCACC }\end{array}$ & & 2 & 2 & 1.00 & 0.73 & 0.69 & 0.60 \\
\hline KU896444 & $\begin{array}{l}\text { F CGTTCAACATCTCCAGAACC } \\
\text { R GAGTACGAGTTCAGCTGTAGCA }\end{array}$ & & 2 & 2 & 1.00 & 0.43 & 0.34 & 0.61 \\
\hline KU896445 & $\begin{array}{l}\text { F AACGTCGGCTGCAACTTT } \\
\text { R GGGCTTGAAAGTGTCGAGAA }\end{array}$ & & 2 & 2 & 1.00 & 0.48 & 0.36 & 0.51 \\
\hline KU896447 & $\begin{array}{l}\text { F GGTGATCAGCTCGAGTCC } \\
\text { R AAATCCACCTCATCTCCAGC }\end{array}$ & & 3 & 3 & 1.00 & 0.50 & 0.37 & 0.46 \\
\hline KU896449 & $\begin{array}{l}\text { F CTCCTCAATCTAAGCCGTCC } \\
\text { R ACGATCACGACTTGCTCTTC }\end{array}$ & & 3 & 2 & 0.67 & 0.53 & 0.44 & 0.66 \\
\hline KU896452 & $\begin{array}{l}\text { F TTCACTTCAGCAGACTTCCC } \\
\text { R GATGCTCCTGGGTTGTTAGA }\end{array}$ & & 3 & 3 & 1.00 & 0.48 & 0.37 & 0.38 \\
\hline KU896454 & $\begin{array}{l}\text { F GCTTGGCTTCAACTCATCTC } \\
\text { R CGGCGGCTTTTATAAGGA }\end{array}$ & & 4 & 4 & 1.00 & 0.28 & 0.25 & 0.37 \\
\hline KU896455 & $\begin{array}{l}\text { F GGAGTGTCTTGTAATAGCCCAC } \\
\text { R CTCCAACCTCCCATTGTTC }\end{array}$ & & 6 & 6 & 1.00 & 0.57 & 0.52 & 0.30 \\
\hline KU896456 & $\begin{array}{l}\text { F GAAACCGTGTTGGTCTTGTC } \\
\text { R AAAGGCCCGATCCAAATC }\end{array}$ & & 3 & 2 & 0.67 & 0.69 & 0.66 & 0.63 \\
\hline KU896457 & $\begin{array}{l}\text { F GACCACCTCGAGAATAAGCA } \\
\text { R CTCCCAGGTAACGTCGAAT }\end{array}$ & & 2 & 2 & 1.00 & 0.50 & 0.37 & 0.53 \\
\hline KU896458 & $\begin{array}{l}\text { F CTCTGGAGAAGCTAAGGAGTGA } \\
\text { R CCATGTTCTCAAACCCTTCC }\end{array}$ & & 2 & 2 & 1.00 & 0.40 & 0.32 & 0.54 \\
\hline KU896459 & $\begin{array}{l}\text { F ACAGCGTGTGGAGGTTCATA } \\
\text { R AGCAGCCACCGTCTAAAAG }\end{array}$ & & 2 & 2 & 1.00 & 0.40 & 0.32 & 0.51 \\
\hline KU896461 & $\begin{array}{l}\text { F GGCAGGATATTCGACGGT } \\
\text { R GGAGATGGTTCTCCTGTTAAGG }\end{array}$ & & 3 & 3 & 1.00 & 0.36 & 0.29 & 0.49 \\
\hline KU896462 & $\begin{array}{l}\text { F CAAGGCTTAGGTCGTAGGTATC } \\
\text { R AAGAGAAGCCAAGATCAGCC }\end{array}$ & & 4 & 3 & 0.75 & 0.62 & 0.54 & 0.45 \\
\hline KU896463 & $\begin{array}{l}\text { F CCATGGTCATATTGCTCTCC } \\
\text { R TGTACTACTCTCTCTCTGCTCTCC }\end{array}$ & & 7 & 7 & 1.00 & 0.52 & 0.41 & 0.28 \\
\hline KU896464 & $\begin{array}{l}\text { F CCAGGAATCTATTGTCGGG } \\
\text { R CAATAATTCAGCCCTCCCTC }\end{array}$ & & 2 & 2 & 1.00 & 0.83 & 0.81 & 0.56 \\
\hline KU896466 & $\begin{array}{l}\text { F GCTGTTTCTTAACAGGAGCAGG } \\
\text { R GGTGTAGCTCAGGCTGTTGTA }\end{array}$ & & 4 & 4 & 1.00 & 0.40 & 0.32 & 0.35 \\
\hline KU896468 & $\begin{array}{l}\text { F CGGGTCTTACGTTCCCTAGTA } \\
\text { R CCAGCTCCATTGATCTTTCC }\end{array}$ & & 3 & 3 & 1.00 & 0.33 & 0.31 & 0.48 \\
\hline KU896471 & $\begin{array}{l}\text { F GGATCGAAACAACCCAGTC } \\
\text { R TCAACCAAACCCAACTCC }\end{array}$ & & 3 & 2 & 0.67 & 0.59 & 0.52 & 0.60 \\
\hline KU896473 & $\begin{array}{l}\text { F ATCGCAGGATCTCTCCAAG } \\
\text { R GATGGATTACTTCCCCTAGGAC }\end{array}$ & & 2 & 2 & 1.00 & 0.50 & 0.39 & 0.59 \\
\hline KU896476 & $\begin{array}{l}\text { F CTCTCTTCTССТCAAACACCC } \\
\text { R GGGTAAGAAAAGGGCAGACA }\end{array}$ & & 4 & 4 & 1.00 & 0.46 & 0.35 & 0.34 \\
\hline KU896477 & $\begin{array}{l}\text { F CTCATCCTCTGCACTTCCAT } \\
\text { R GGCAATTGCATCGTCAAG }\end{array}$ & & 2 & 2 & 1.00 & 0.38 & 0.33 & 0.46 \\
\hline KU896478 & $\begin{array}{l}\text { F GTCTTGGGAGTGGCTTTTGT } \\
\text { R CTCATCATTTACCTCCGACG }\end{array}$ & & 2 & 2 & 1.00 & 0.28 & 0.24 & 0.58 \\
\hline Mean & & & 2.90 & 2.75 & 0.94 & 0.44 & 0.37 & 0.45 \\
\hline
\end{tabular}

NA: Number of alleles, ${ }^{\mathrm{y}} \mathrm{NPA}$ : Number of polymorphic alleles, ${ }^{\mathrm{x}} \mathrm{FPM}$ : Fraction of polymorphic marker, ${ }^{\mathrm{w}}$ GD: Gene diversity, ${ }^{\mathrm{v}}$ PIC: Polymorphism information content, "SI: Shannon's Information index. 
Table 6 Association analyses between SSR markers and morphological characteristics and phenolic compound contents of the kenaf genotypes

\begin{tabular}{|c|c|c|c|c|c|}
\hline Marker & Trait & $\begin{array}{l}\mathrm{Q} \text { GML } \\
P \text {-value }\end{array}$ & $\mathrm{R}^{2}$ & $\begin{array}{c}\mathrm{Q}+\mathrm{K} \text { MLM } \\
P \text {-value }\end{array}$ & $\mathrm{R}^{2}$ \\
\hline KU896377 & Vein color & $*$ & 0.266 & $*$ & 0.248 \\
\hline KU896382 & Total polyphenol & $*$ & 0.123 & $*$ & 0.131 \\
\hline KU896384 & Petal color & $* * *$ & 0.142 & $*$ & 0.363 \\
\hline KU896387 & Leaf color & $* * *$ & 0.303 & $*$ & 0.247 \\
\hline KU896399 & Leaf color & $*$ & 0.203 & $*$ & 0.231 \\
\hline KU896408 & Stem color & $*$ & 0.166 & $*$ & 0.187 \\
\hline KU896423 & Vein color & $*$ & 0.258 & $*$ & 0.233 \\
\hline KU896426 & KGRI & $* *$ & 0.159 & $*$ & 0.160 \\
\hline KU896438 & Petal color & $* * *$ & 0.140 & $*$ & 0.309 \\
\hline KU896440 & Petal color & $* * *$ & 0.206 & $*$ & 0.246 \\
\hline KU896441 & Leaf color & $* * *$ & 0.973 & $* *$ & 0.999 \\
\hline KU896447 & Leaf shape & $* *$ & 0.235 & $*$ & 0.181 \\
\hline KU896449 & Leaf color & $* * *$ & 0.974 & $* * *$ & 0.999 \\
\hline KU896449 & Corolla color & $* * *$ & 0.172 & $*$ & 0.289 \\
\hline KU896449 & Stem color & $*$ & 0.219 & $*$ & 0.248 \\
\hline
\end{tabular}

(Cheng et al. 2004; Chen et al. 2011; Zhang et al. 2013). In additions, these results are higher than the findings of $\mathrm{Li}$ et al. (2016) in 28 kenaf cultivars using 72 EST-SSR markers. Especially, the maximum values of GD and PIC occurred with the KU896464 and the maximum values of SI occurred with KU896449. This suggests that KU896464 and KU896449 markers could be used to assess genetic diversity in genotypes.

The correlation analysis showed flowering time and DM yield, seed yield and phenolic compounds were significantly correlated. Also, comparison of three phylogenetic tree based on morphological and chemical characteristics with those based on SSR data showed that the groups formed by the six early flowering genotypes (C9, C11, C14, C15, C17, C19) grouped similarly in SSR phylogenetic tree. Ultimately, early flowering, mid-late flowering and non-flowering groups were clearly divided by genotypes in SSR phylogenetic tree. Similarly, Jeong et al. (2017) reported that phylogenetic and population structure showed that the 45 accessions could be clearly divided into three groups based on different days to flowering by EST-SSR markers through de novo RNA sequencing. The flowering period has been reported to be an indication of sensitivity of kenaf genotypes to photoperiod, later flowering genotypes being photo-insensitive (Webber et al. 2002). Korean Kenaf cultivars are divided into three maturation groups depending on flowering date; early-flowering, mid-late flowering, and late flowering (Kang et al., 2016). Earlyflowering cultivars bloom in $70 \sim 80$ day after sowing. Such varieties allow seed harvested in Korea, but the brief vegetative growth period produces shorter plants of lower biomass (Webber and Bledsoe, 2002; Kang et al., 2016). Late-flowering cultivar grows vegetative for $130 \sim 140$ days and yield significantly higher biomass. However, late flowering increases the risk of seed shattering (Kang et al., 2016). To achieve profitability, the selection of the best cultivar for each country is important. The breeding of kenaf cultivars for yield and economic important with adaptation to local conditions has been conducted actively using these genetic resources (Alexopoulou et al., 2013; Kang et al., 2016). Breeding selection of kenaf starts mostly from the introduction of new cultivars, and the breeding selection of new cultivars is the most effective measure for increasing yield and improving functional quality (Ryu et al., 2016; Jeong et al., 2017). The Jangdae and Jeokbong cultivars were derived by gamma ray (300 Gy) treatment. The mid-late cultivar Jangdae, which affords both high biomass and high seed yield, has been registered in Korea (Kang et al., 2016). The Jeokbong cultivar has distinctive morphological characteristics such as dark purple color. The low levels of phenolic compounds observed in the present study for the Jeokbong cultivar, but their antioxidant and antioxidant and ACE inhibition activity are approximately $4 \sim 5$ times higher than other cultivars caused by anthocyanin (Ryu et al., 2017b). Based on the flowering time, the morphological and chemical phylogenetic tree revealed an unclear pattern of division between mutant cultivars (Jangdae, Baekma and Jeokbong) and these original genotypes (Jinju and C14), while the genetic phylogenetic tree showed that the mutant cultivars grouped with the those original genotypes (Jinju and C14). Thus, these results could be used for the selection of kenaf cultivars with improved yield and functional compounds. Our results of morphological, chemical 
and genetic phylogenetic trees were no related with the origin. In previous studies on the origin, many studies agree that kenaf originated from Africa (Dempsey 1975; Alexopoulou et al. 2013). However, the knowledge of how kenaf was introduced in Asia is limited but it is known that it came from Africa (Dempsey 1975; Alexopoulou et al. 2013; Zhang et al. 2013). In additions, it is likely that cluster analysis using country of origin was not able to differentiate among all cultivars because of the limited number of accessions. The SSR phylogenetic tree clearly showed that the genotypes divided into flowering time, but the genomic and EST SSR markers not significant correlated with flowering time. Marker assisted selection has the potential to improve the efficiency of plant breeding because of its increased accuracy and liability. However, with this large average distance, greater saturation would be needed for practical application especially for marker assisted selection (Varshney et al. 2005; Varshney and Tuberosa, 2007; Zhang et al. 2011). To the best of our knowledge, this is the first report on the correlation of molecular markers with phenolic compounds, morphology and agronomic traits in kenaf. These 15 SSR markers could be used for selection of kenaf cultivars with improved morphological characteristics and functional compounds (total polyphenol and KGRI). The mapping of genes controlling agronomic traits and functional compounds coupled with the widespread availability of easy to use simple sequence repeat (SSR) markers (Varshney et al. 2005; Varshney and Tuberosa, 2007). These results could be used for the selection of kenaf cultivars with improved yield and may be a good candidate for pharmaceutical products.

Acknowledgements: This work was supported by grants from the Nuclear R\&D Program by the Ministry of Science and ICT (MSIT), and the research program of KAERI, Republic of Korea.

\section{Refernces}

Alexopoulou E, Papattheohari Y, Christou M, Monti A (2013) Keanf: A multi-purpose crop for sever industrial applications. Springer-verlag, London. pp.1-15

Cazarolli LH, Zanatta L, Jorge AP, De SE, Horst H, Woehl VM, Pizzolatti MG, Szpoganicz B, Silva FR (2006) Follow-up studies on glycosylated flavonoids and their complexes with vanadium: their anti-hyperglycemic potential role in diabetes. Chem Biol Interact 163:177-191

Chen M, Wei C, Qi J, Chen X, Su J, Li A, Tao A, Wu W (2011) Genetic linkage map construction for kenaf using SRAP, ISSR and RAPD markers. Plant Breed 130:79-87

Cheng Z, Lu BR, Sameshima K, Fu DX, Chen JK (2004)
Identification and genetic relationships of kenaf (Hibiscus cannabinus L.) germplasm revealed by AFLP analysis. Genet Resour Crop Evol 51:393-401

Dempsey JM (1975) Fiber Crops. The University Presses of Florida, Gainesville, USA.

Faruq G, Alamgir MA, Rahman MM, Motior MR, Zakaria HP, Marchalina B, Mohamed NA(2013) Morphological Characterization of kenaf (Hibiscus cannabinus L.) in Malaysian tropical environment using multivariate analysis. J Animal Plant Sci 23:60-67

IAEA (2016) Mutant variety database (MVD). http://mvgs.iaea.org/ AboutMutantVarieties.aspx/

Jeong SW, Kwon SJ, Ryu J, Kim JB, Ahn JW, Kim SH, Jo YD, Choi HI, Im SB, Kang SY (2017) Development of EST-SSR markers through de novo RNA sequencing and application for biomass productivity in kenaf (Hibiscus cannabinus L.) Genes Genom DOI 10.1007/s13258-017-0582-z

Jin CW, Ghimeray AK, Wang L, Xu ML, Piao JP, Cho DH (2013) Far infrared assisted kenaf leaf tea preparation and its effect on phenolic compounds, antioxidant and ACE inhibitory activity. J Med Plants Res 7:1121-1128

Jorge AP, Horst H, Sousa E, Pizzolatti MG, Silva FRMB (2004) Insulinomimetic effects of kaempferitrin on glycaemia and on ${ }^{14} \mathrm{C}$ glucose uptake in rat soleus muscle. Chem Biol Inter 149:89-96

Kang SY, Kwon SJ, Jeong SW, Kim JB, Kim SH, Ryu J (2016) An improved kenaf cultivar 'Jangdae' with seed harvesting in Korea. Korean J Breed Sci 48:349-354

Kobaisy M, Tellez MR, Webber CL, Dayan FE, Schrader KK, Wedge DE (2001) Phytotoxic and fungitoxic activities of the essential oil of kenaf (Hibiscus cannabinus L.) leaves and its composition. J Agric Food Chem 49:3768-3771

Li HL, Chen A, Tang H, Li J, Huang S (2016) Characterization of the kenaf (Hibiscus cannabinus) global transcriptome using illumina paired-end sequencing and development of EST-SSR markers. PLoS ONE 11(3): e0150548

Ryu J, Ha BK, Kim DS, Kim JB, Kim SH, Kang SY (2013) Assessment of growth and seed oil composition of kenaf (Hibiscus cannabinus L.) germplasm. J Crop Sci Biotech 16: 297-302

Ryu, J, Kwon SJ, Ahn JW, Ha BK, Jeong SW, Im SB, Kim JB, Kim SH, Lee YK, Kang SY (2016a) Evaluation of nutritive value and identification of fungi in silage from new kenaf (Hibiscus cannabinus) cultivars. Int J Agric Biol 18:1159-1168

Ryu J, Kwon SJ, Ahn JW, Jo YD, Kim SH, Jeong SW, Lee MK, Kim JB, Kang SY (2017a) Phytochemicals and antioxidant activity in the kenaf plant (Hibiscus cannabinus L.) J Plant Biotechnol 44:191-202

Ryu J, Kwon SJ, Ahn JW, Kim SH, Lee SY, Kim JB, Jo YD, Ha BK, Kang SY. (2017b) Development of stem-color mutant kenaf (Hibiscus cannabinus) cultivar 'Jeokbong' and analysis of functional compounds. Hortic Sci Technol (in press)

Ryu, J, Kwon SJ, Jo YD, Jin CH, Nam BM, Lee SY, Jeong SW, Im SB, Oh SC, Cho L, Ha BK, Kang SY (2016b) Comparison of phytochemicals and antioxidant activity in blackberry (Rubus 
fruticosus L.) fruits of mutant lines at the different harvest time. Plant Breed Biotech 4:242-251

Saitou N, Nei M (1987) The neighbor-joining method: a new method for reconstructing phylogenetic trees. Mol Biol Evol. 1987 Jul;4:406-425

Taylor CS, Kugler DE (1992) Kenaf: annual fiber crop products generate a growing response from industry. In: New Crops, New Uses, New Markets, 1992 Yearbook of Agriculture. USDA, Washington, D.C., pp 92-98

Thakur AK, Singh KH, Singh L, Nanjundan J, Khan YJ, Singh D (2017) Patterns of subspecies genetic diversity among oilseed Brassica rapa as revealed by agro-morphological traits and SSR markers. J Plant Biochem Biotechnol 26:282-292

Varshney RK, Graner A, Sorrells ME (2005) Genic microsatellite markers in plants: features and applications. Trends Biotechnol 23:48-55

Varshney RK, Tuberosa R (2007) Genomics-assisted crop im- provement. Springer Dordrecht, Netherlands.

Visser T, Verhaegh JJ, De Vries D (1971) Pre-selection of compact mutants induced by X-ray treatment in apple and pear. Euphytica 20:195-207

Zhang L, Li A, Wang X, Zhang G, Su J, Qi J, Guan C (2013) Genetic diversity of kenaf (Hibiscus cannabinus) evaluated by inter-simple sequence repeat (ISSR) Biochem Genet 51:800810

Zhang L, Wan X, Xu J, Lin L, Qi J (2015) De novo assembly of kenaf (Hibiscus cannabinus) transcriptome using Illumina sequencing for gene discovery and marker identification. Mol Breed 35:1-11

Zhao S, Li X, Cho DH, Arasu MV, Al-Dhabi NA, Park SU (2014) Accumlation of kaempferitrin and expression of phenylpropanoid biosynthetic genes in kenaf (Hibiscus cannabinus). Molecules 19:16987-16997 